CIUDAD Y TERRITORIO

ESTUDIOS TERRITORIALES

ISSN(P): 2697-231X; ISSN(E): 2697-2328

Vol. LIII, N.ำ Monográfico 2021

Págs. 179-200

https://doi.org/10.37230/CyTET.2021.M21.10

CC BY-NC-ND

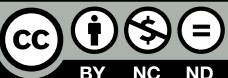

\title{
Los Catálogos de Barrios Vulnerables de España. Análisis de la vulnerabilidad en las ciudades españolas entre 1991 y 2011
}

\author{
Iván RodRígUEZ-SUÁREZ ${ }^{(1)}$ \\ Agustín HERNÁNDEZ-AJA ${ }^{(2)}$ \\ José Manuel GómEZ-GIMÉNEZZ ${ }^{(3)}$ \\ Ángela MateSANZ-PARELLADA ${ }^{(4)}$ \\ Ana Díez-Bermejo(5)
}

${ }^{(1)}$ Arquitecto. Investigador y profesor asociado (2)Dr. Arquitecto. Catedrático

${ }^{3}$ Arquitecto y graduado en Ciencia Política y Administración. Investigador ${ }^{(4)}$ Dra. Arquitecta. Profesora asociada ${ }^{(5)}$ Arquitecta. Investigadora Todos ellos del Departamento de Urbanística y Ordenación del Territorio Universidad Politécnica de Madrid

Resumen: Los Catálogos de Barrios Vulnerables de España de 1991, 2001 y 2011 examinan la vulnerabilidad urbana en las ciudades españolas mayores de 50 mil habitantes y capitales de provincia. Su principal conclusión es que durante las dos décadas analizadas los desequilibrios internos de las ciudades españolas aumentaron de forma significativa y continuada, en un doble proceso de incremento y concentración espacial de población vulnerable, independiente de las coyunturas económicas y políticas. Los Catálogos son uno de los componentes principales del Observatorio de la Vulnerabilidad Urbana del actual Ministerio de Transportes, Movilidad y Agenda Urbana. Precisamente, la Agenda Urbana Española los propone como herramientas para la consecución de dos de sus objetivos: impulsar la regeneración urbana y fomentar la cohesión social buscando la equidad.

Recibido: 19.10.2020; Revisado: 26.10.2020

Correo electrónico: ivan.rsuarez@upm.es; N.º ORCID: https://orcid.Org/0000-0002-9139-0280

Correo electrónico: agustin.hernandez@upm.es; N. ORCID: https://orcid.Org/0000-0002-4485-058x

Correo electrónico: jm.gomez@upm.es; N. ${ }^{\circ}$ ORCID: https://orcid.Org/0000-0003-4513-0725

Correo electrónico: angela.matesanz@upm.es; N. ORCID: https://orcid.Org/0000-0002-1670-1296

Correo electrónico: ana.diez@upm.es; N. ${ }^{\circ}$ ORCID: https://orcid.Org/0000-0001-8524-75

Los autores agradecen los comentarios y sugerencias realizados por los evaluadores anónimos, que han contribuido a mejorar y enriquecer el manuscrito original. 


\title{
Spain's Urban Analyses of Vulnerable Neighbourhoods between 1991 and 2011
}

\begin{abstract}
Spain's Urban Analyses of Vulnerable Neighbourhoods in 1991, 2001 and 2011 examine urban vulnerability in the Spanish cities with more than fifty thousand inhabitants and provincial capitals. Its main conclusion is that the internal imbalances in the Spanish cities increased significantly and continuously during the two decades analysed, in a double process of expansion and spatial concentration of vulnerable population, regardless of market condition and political juncture. The Urban Analyses are one of the main components of The Observatory of Urban Vulnerability from the current Spanish Ministry of Transport, Mobility and Urban Agenda. Indeed, the Spanish Urban Agenda indicates it as a tool to achieve two of its goals: promoting urban regeneration and fostering social cohesion to seek equity.
\end{abstract}

KEYWORDS: Urban vulnerability; Vulnerable neighbourhoods; Observatory of Urban Vulnerability; Deprived neighbourhoods; Urban inequality.

\section{Introducción. El Observatorio de la Vulnerabilidad Urbana}

I Observatorio de la Vulnerabilidad Urbana (OVU) es un proyecto a largo plazo del Ministerio de Transportes, Movilidad y Agenda Urbana iniciado en 2009 y en actualización permanente. Las investigaciones y trabajos desarrollados en el marco del OVU sirven como punto de partida para el diseño de las políticas urbanas que entran dentro de las competencias del Ministerio y atienden al mandato de la Disposición Adicional Primera del Real Decreto Legislativo $7 / 2015$, por el que se aprueba el Texto Refundido de la Ley del Suelo y Rehabilitación Urbana (MINISTERIO DE FOMENTO, 2015), que regula el Sistema de Información Urbana al servicio de las políticas públicas para un medio urbano sostenible. Entre otros instrumentos, la Ley prevé la elaboración de:

"mapas de ámbitos urbanos deteriorados, obsoletos, desfavorecidos o en dificultades, precisados de regeneración y renovación urbanas, o de actuaciones de rehabilitación edificatoria".

La previsión de este tipo de información destinada específicamente al análisis de ámbitos vulnerables se introdujo por la Ley 8/2013 de rehabilitación, regeneración y renovación urbanas (JEFATURA DEL ESTADO, 2013a: Disposición Adicional Primera b), aunque el OVU existía ya formalmente desde el año 2009.
Los contenidos elaborados para el OVU se encuentran disponibles al público en una sección específica dentro de la página electrónica del Ministerio ${ }^{1}$ y son los siguientes:

- Atlas de la Vulnerabilidad Urbana y Atlas de la Edificación Residencial, organizados en dos visualizadores con información a nivel de sección censal para todos los municipios españoles y datos obtenidos de los Censos de Población y Vivienda de 2001 y 2011.

- Análisis de las características de la Edificación Residencial en España 2001 y 2011 (HERNÁNDEZ \& al., 2013 y 2014). Se trata de dos informes a nivel nacional y por comunidades autónomas sobre las características del parque residencial y sus necesidades de rehabilitación, elaborados a partir de los Censos de 2001 y 2011.

- Análisis Urbanístico de Barrios Vulnerables de España, referido a los años 1991, 2001, 2006 y 2011, denominado también Catálogo de Barrios Vulnerables, en el que se analiza la vulnerabilidad urbana de las ciudades españolas mayores de 50 mil habitantes y capitales de provincia para delimitaciones de escala de barrio elaboradas exprofeso. Los tres catálogos fueron elaborados a partir de datos de los Censos de 1991, 2001 y 2011 y sus principales resultados pueden ser consultados en el Visor del Catálogo de Barrios Vulnerables.

\footnotetext{
1 https://www.mitma.gob.es/arquitectura-vivienda-y-suelo/urbanismo-y-politica-de-suelo/observatorio-de-la-vulnerabilidad-urbana
} 
- Publicaciones derivadas de los Catálogos de Barrios Vulnerables: Atlas de Barrios Vulnerables de España: 12 Ciudades 19912001-2006 (HERNÁNDEZ \& al., 2015); Barrios Vulnerables de las grandes ciudades españolas 1991-2001-2011 (HERNÁNDEZ \& al., 2018b); y Vulnerabilidad residencial en las grandes ciudades españolas 2001-2011 (HERNÁNDEZ \& al., 2018c).

- Otros estudios e informes: Informe sobre Fórmulas innovadoras de Gestión y Financiación en actuaciones de Regeneración de Barrios (HERNÁNDEZ \& al., 2018a); Mapa sobre Vivienda y Comunidad Gitana en España 2007.

- Enlaces a otros Observatorios europeos, autonómicos o municipales.

La necesidad de identificar los "barrios o entornos urbanos que presenten un mayor grado de vulnerabilidad social, económica y ambiental como áreas de actuación preferente para favorecer la equidad en el desarrollo urbano y luchar contra las bolsas de pobreza, la exclusión social y la desigualdad" es una de las líneas de actuación previstas por la Agenda Urbana Española (MINISTERIO DE FOMENTO, 2019) dentro del objetivo específico 6.1 centrado en reducir el riesgo de pobreza y exclusión en entornos urbanos desfavorecidos. La Agenda Urbana Española, en línea con la Agenda Urbana Europea y con la consolidación de los organismos o instrumentos que detectan, analizan o hacen seguimiento de áreas con condiciones de vulnerabilidad en el contexto europeo, refiere los Observatorios de Barrios Vulnerables como herramientas útiles a estos efectos y la idoneidad de aplicar sobre ellos instrumentos de carácter integrado o integral. Los Catálogos de Barrios Vulnerables del OVU se proponen, además, como fuente de indicadores de seguimiento y evaluación de los objetivos 2.5 Impulsar la regeneración urbana y 6.1 Fomentar la cohesión social y buscar la equidad.

El objeto de este artículo es analizar y presentar los principales resultados de los tres Catálogos de Barrios Vulnerables de España de 1991, 2001 y 2011, como producto más longevo del OVU y uno de sus componentes principales (DE SANTIAGO, 2018) y de utilidad para el desarrollo de la Agenda Urbana Española. Se realiza en primer lugar un recorrido histórico que contextualiza y explica cómo se elaboraron los tres

\footnotetext{
2 La denominación utilizada en el primer Catálogo era "barrios desfavorecidos". En el catálogo de 2001 se sustituyó por la denominación de "barrios vulnerables" al considerarse que se ajusta mejor a la realidad de los ámbitos delimitados. El término vulnerable indica una posible situación crítica y la
}

Catálogos y la metodología utilizada. A continuación, se exponen los principales resultados agregados de los tres Catálogos, así como las herramientas y productos de difusión de la información. El artículo se cierra con unas conclusiones en las que se analizan las principales ventajas y limitaciones de los Catálogos y sus perspectivas de futuro.

\section{Los Catálogos de Barrios Vulnerables}

Los Catálogos de Barrios Vulnerables de España de 1991, 2001 y 2011 fueron desarrollados entre 1996 y 2017 mediante convenios de colaboración entre el Gobierno de España, a través del Ministerio competente en cada momento en materia de vivienda y políticas urbanas, y el Instituto Juan de Herrera (IJH) de la Universidad Politécnica de Madrid. En el primer Catálogo de 1991 se contó con la asistencia técnica al Ministerio para el tratamiento de los datos por parte de la empresa Analística, y en los de 2001 y 2011 de Argea Consultores. Los datos estadísticos utilizados fueron facilitados por el Instituto Nacional de Estadística (INE).

El objetivo de los Catálogos es localizar aquellos barrios en los que las condiciones de la población residente sean sensiblemente peores que las del conjunto de la población española. Los Catálogos constituyen una reflexión sobre la vulnerabilidad urbana desde una perspectiva urbanística, centrada en la determinación y análisis de ámbitos concretos que se encuentran en peor situación que el resto de los tejidos urbanos españoles, y que pueden constituir piezas que por su tamaño, autonomía y homogeneidad puedan ser objeto de planes y/o programas de intervención que mejoren su posición. Los Catálogos ofrecen, además, una panorámica de la vulnerabilidad urbana de las ciudades españolas a lo largo de las dos décadas que abarcan, permitiendo analizar tanto los desequilibrios existentes en una ciudad, región o todo el país, como su evolución en el periodo.

El primer Catálogo de Barrios Vulnerables de $1991^{2}$ se realizó en 1996 en el marco de un proyecto desarrollado en el seno de la Organización para la Cooperación y el Desarrollo Económicos (OCDE, 1998), del que España formaba parte,

necesidad de intervenir mediante políticas integrales para evitar la degradación social y funcional del área. Por su parte, el término desfavorecido puede interpretarse tan sólo en cuanto a carencias materiales, lo que conllevaría intervenciones sectoriales al uso. 
y que tenía como objetivo estudiar la situación de los "barrios desfavorecidos". El proyecto contemplaba la redacción de un informe por el Ministerio de Obras Públicas Transportes y Medio Ambiente con el análisis de las características de estos barrios y las medidas que los gobiernos nacionales deberían desarrollar para la integración de políticas públicas en los mismos. La metodología de la OCDE preveía el análisis de dos de las dimensiones de la vulnerabilidad, paro y renta, en ámbitos de entre 5.000 y 10.000 habitantes de ciudades mayores de 100 mil habitantes. En el planteamiento metodológico del Catálogo, y ante la ausencia de información estadística sobre renta en el caso español, se optó por utilizar dos indicadores proxy para este indicador concreto, el nivel de estudios y las carencias en el alojamiento, tras comprobar su alto grado de correlación con el nivel de renta. También se amplió la horquilla del tamaño de los ámbitos a entre 3.500 habitantes y 15.000 habitantes, y el cuerpo de estudio a las ciudades mayores de 50 mil habitantes y capitales de provincia. Fruto de este primer trabajo se publicó el libro dirigido por Félix Arias Goytre: "La desigualdad urbana en España" (ARIAS, 2000).

Pasados doce años, en el año 2008, el entonces Ministerio de Vivienda retomó la idea de actualizar el Catálogo con los datos del Censo de 2001 replicando la metodología de trabajo del Catálogo de 1991. Puesto que ya habían pasado ocho años desde la fecha de referencia del Censo de $2001^{3}$ y la realidad del país había cambiado sustancialmente debido a una llegada considerable de población inmigrante, se decidió que sería necesario realizar también un estudio adicional que analizase dónde se localizaban estas nuevas poblaciones extranjeras al objeto de comprobar si lo hacían en los barrios catalogados como vulnerables o no. Esto dio lugar a la elaboración de una Adenda, con datos del Padrón de 2006, en la que se analizaban precisamente estas concentraciones de población extranjera, para comprobar su correspondencia con los perímetros de los barrios vulnerables delimitados. Los trabajos del Catálogo de 2001 se desarrollaron durante tres años: 2008, en el que se definió la metodología; 2009 , en el que analizaron las 20 ciudades con mayor población vulnerable; y 2010, en el que se analizaron las restantes ciudades incluidas en el estudio.

La metodología de los dos primeros Catálogos, de 1991 y 2001, es la misma, con variaciones y ajustes tan sólo en la definición de algunos de los indicadores, en función de la información disponible en cada Censo, y la ampliación de los contenidos elaborados en el Catálogo de 2001, que incorporan información más detallada para cada una de las áreas, así como informes específicos de análisis de cada comunidad autónoma y ciudad. La metodología incluía trabajo de gabinete y de campo, con la visita a cada una de las ciudades analizadas en las que se hubiesen detectado posibles barrios vulnerables mediante análisis estadístico, para su verificación con los servicios técnicos municipales. Ambos Catálogos son anteriores a la creación del OVU en el año 2009 y fueron integrados en el mismo desde su fundación.

El entonces Ministerio de Fomento propuso el inicio de los trabajos del Catálogo de 2011 en el año 2013 y su elaboración se acometió en tres etapas. En 2013 se definió la metodología, que tuvo que ser revisada en profundidad respecto a la utilizada para los datos de 2001 por cambios en la elaboración y disponibilidad de información del Censo de 2011. Si los dos censos anteriores, de 1991 y 2001, se basaban en un trabajo de campo universal, que permitía disponer de la información para todas las secciones censales; el Censo de 2011 se basó en una muestra, lo que impedía disponer de algunas de las variables en la escala de la sección censal. Además, en la última edición del catálogo se prescindió del trabajo de campo y toda la investigación se realizó en gabinete, con la plena incorporación de Sistemas de Información Geográfica (SIG) y gestión de bases de datos que, en los trabajos anteriores, especialmente en el primero, se encontraban aún en fase embrionaria.

En 2015, se elaboró la base de datos de indicadores para todo el cuerpo de estudio y se comprobó si los barrios vulnerables delimitados en 2001 continuaban siendo vulnerables o no. Finalmente, en el año 2016 se delimitaron las nuevas áreas estadísticas vulnerables no coincidentes con los barrios vulnerables delimitados en 2001, así como las áreas con concentraciones significativas de población extranjera, sin que se realizase el trabajo de delimitación urbanística que permitiese denominarlas barrios.

Los Catálogos se complementan con los datos ofrecidos en los Atlas de Vulnerabilidad Urbana de 2001 y 2011, que ofrecen indicadores adicionales a nivel de sección censal para todos los municipios españoles, aunque sin delimitar áreas que superen esta escala.

\footnotetext{
${ }^{3}$ Los resultados definitivos del Censo 2001 fueron publicados en febrero de 2004.
} 
Los Catálogos de 2001 y 2011 dieron lugar a tres publicaciones derivadas en las que se analiza y presenta en profundidad la evolución de la vulnerabilidad urbana en las ciudades españolas mayores de 300 mil habitantes del Catálogo de 2001 (HERNÁNDEZ \& al., 2015) y de 2011 (HERNÁNDEZ \& al., 2018b y 2018c), 12 y 13 ciudades respectivamente (FIG. 1).

Los tres Catálogos, y especialmente los dos primeros, al incluir trabajo de campo que permitió conocer las ciudades analizadas y establecer contacto con los servicios técnicos municipales, constituyeron un laboratorio de formación de urbanistas en la Escuela Técnica Superior de Arquitectura de Madrid, al requerir un trabajo intensivo desarrollado a lo largo de varios años por un grupo amplio de estudiantes, arquitectos recién titulados y profesores. Algunos de los miembros del equipo han participado en la elaboración de más de uno de los Catálogos, lo que, unido a la dirección en los tres Catálogos de Agustín Hernández Aja, por parte de la Universidad Politécnica, y en los dos últimos, de Eduardo de Santiago Rodríguez, por parte del Ministerio, ha permitido dotarlos de continuidad y coherencia, salvando las posibles limitaciones derivadas de la metodología y medios utilizados y de su dilación discontinuada a lo largo de dos décadas.

\subsection{Metodología}

\subsection{1. Ámbito de estudio}

Los Catálogos de 1991, 2001 y 2011 se realizaron para las ciudades mayores de 50 mil habitantes y capitales de provincia. Los trabajos del Catálogo de 2001 fueron iniciados en 2008. La antigüedad de los datos y los intensos cambios sociodemográficos que se habían producido desde 2001, hicieron que se escogiera 2006 como fecha de referencia para la población mayor de 50 mil habitantes. De esta forma el cuerpo de estudio se ajustó en cada Catálogo en función de las variaciones de la población en los municipios. Las ciudades estudiadas fueron 116 en el Catálogo de 1991, 139 en el de 2001 y 147 en el de 2011. Y la población estudiada pasó de 20 millones de habitantes en 1991 a 24,5 millones en 2011, lo que supuso incluir a más de la mitad de la población española (FIG. 2).

Cinco comunidades concentran la mayor parte del cuerpo de estudio. Estas cinco comunidades, de mayor a menor población estudiada, son: Comunidad de Madrid, Andalucía, Cataluña, Comunitat Valenciana y Castilla y León, con 20, 29, 23, 15 y 10 ciudades respectivamente en 2011 , suponiendo el $57,6 \%$ de las ciudades y un $69,7 \%$ de la población estudiada. La ampliación del cuerpo de estudio de los Catálogos se produjo en su práctica totalidad en estas comunidades autónomas, a excepción de Castilla y León, en la que no se incrementaron las ciudades estudiadas y apenas lo hizo la población. La Región de Murcia también aumento de forma significativa su población e incorporó una ciudad, Molina de Segura, en el último Catálogo.

Como norma general, en la mayoría de las Comunidades Autónomas, el número de ciudades estudiadas aumentó (en seis de ellas) o se mantuvo constante (en nueve de ellas). Sin embargo, cuatro de las ciudades analizadas en 1991 dejaron de formar parte de los Catálogos al situarse por debajo de los 50 mil habitantes en 2001, todas ellas localizadas en la cornisa
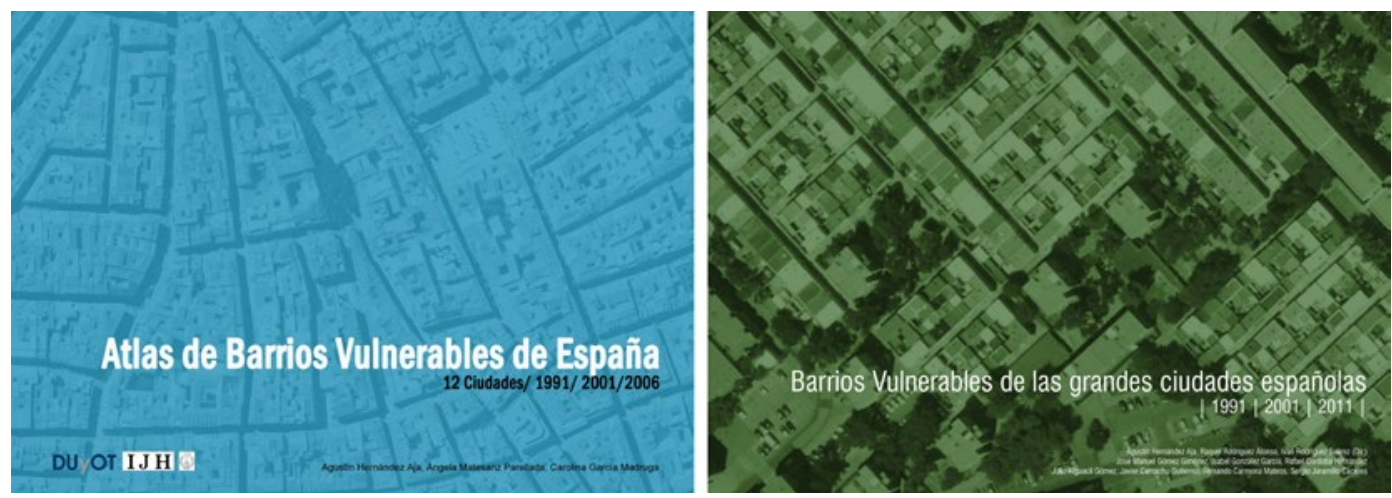

FIG. 1/ Portada de dos de las publicaciones derivadas de los Catálogos de Barrios Vulnerables de 1991, 2001 y 2011 en las que se analiza la vulnerabilidad en las ciudades mayores de 300 mil habitantes.

Fuente: HERNÁNDEZ \& al. (2015 y 2018b). 
cantábrica y afectadas por la reconversión industrial de los años 90 del siglo XX: Basauri y Santurce en el País Vasco, y Mieres y Langreo en Asturias (aunque en esta última comunidad se incorporó una ciudad en el catálogo de 2011, Siero).

\subsubsection{Fuentes de datos y delimitaciones espaciales}

La fuente de datos de los Catálogos son los Censos de Población y Vivienda de los años 1991, 2001 y 2011. Para la Adenda de población extranjera de 2006 se utilizaron datos del Padrón de 2006. Las variables utilizadas para el cálculo de indicadores se obtienen en la máxima desagregación espacial disponible: la sección censal.

A pesar de las limitaciones que puedan presentar, los Censos son la única fuente disponible en España que permite realizar un trabajo como el de los Catálogos, al ofrecer información para todo el estado y con un nivel de desagregación espacial suficiente (la sección censal) como para abordar el análisis y delimitación urbanística de los barrios.

Los Catálogos se basan en la utilización de dos delimitaciones espaciales: el Área Estadística Vulnerable (AEV) y el Barrio Vulnerable (BV). El $A E V$ es una delimitación estadística que agrupa diversas secciones censales con población comprendida entre 3.500 y 15.000 habitantes $^{4}$,

\begin{tabular}{|c|c|c|c|c|c|c|c|c|c|}
\hline \multirow[b]{2}{*}{ CCAA } & \multicolumn{3}{|c|}{1991} & \multicolumn{3}{|c|}{2001} & \multicolumn{3}{|c|}{2011} \\
\hline & $\begin{array}{c}\text { N.o } \\
\text { MUN }\end{array}$ & POB. EST. & $\begin{array}{l}\text { \%POB. } \\
\text { EST. }\end{array}$ & $\begin{array}{l}\text { N.o } \\
\text { MUN }\end{array}$ & POB. EST. & $\begin{array}{c}\text { \%POB. } \\
\text { EST. }\end{array}$ & $\begin{array}{l}\text { N.o } \\
\text { MUN }\end{array}$ & POB. EST. & $\begin{array}{c}\text { \%POB. } \\
\text { EST. }\end{array}$ \\
\hline Madrid, C. de & 12 & 4.315 .472 & 87,2 & 17 & 4.683 .470 & 86,4 & 20 & 5.403 .367 & 84,6 \\
\hline Andalucía & 19 & 3.188 .725 & 45,9 & 28 & 3.693 .908 & 50,2 & 29 & 4.228 .061 & 50,7 \\
\hline Cataluña & 18 & 3.547 .913 & 58,6 & 23 & 3.632 .022 & 57,3 & 23 & 4.054 .333 & 54,3 \\
\hline C. Valenciana & 9 & 1.624 .348 & 42,1 & 13 & 1.861 .334 & 44,7 & 15 & 2.274 .816 & 45,6 \\
\hline Castilla y León & 10 & 1.131 .584 & 44,4 & 10 & 1.116 .099 & 45,4 & 10 & 1.143 .217 & 45,4 \\
\hline Canarias & 4 & 743.300 & 49,8 & 7 & 893.569 & 52,7 & 8 & 1.089 .882 & 52,4 \\
\hline País Vasco & 9 & 1.142 .225 & 54,3 & 7 & 1.029 .631 & 49,4 & 7 & 1.066 .382 & 49,1 \\
\hline Galicia & 7 & 951.405 & 34,8 & 7 & 955.569 & 35,5 & 7 & 995.424 & 36,1 \\
\hline Murcia, Reg. de & 3 & 401.965 & 38,4 & 4 & 679.813 & 56,8 & 4 & 812.044 & 55,7 \\
\hline Aragón & 3 & 667.046 & 56,1 & 3 & 692.306 & 57,5 & 3 & 765.806 & 57,5 \\
\hline Asturias, Pr. de & 5 & 645.661 & 59,0 & 3 & 550.758 & 51,8 & 4 & 637.054 & 59,6 \\
\hline Castilla-La M. & 7 & 469.298 & 28,3 & 7 & 470.525 & 26,7 & 7 & 611.418 & 29,2 \\
\hline Baleares & 1 & 296.754 & 41,8 & 1 & 333.801 & 39,7 & 1 & 402.044 & 36,7 \\
\hline Extremadura & 3 & 246.098 & 23,2 & 3 & 266.506 & 25,2 & 3 & 304.640 & 27,8 \\
\hline Cantabria & 2 & 251.102 & 47,6 & 2 & 236.194 & 44,1 & 2 & 233.220 & 39,6 \\
\hline Navarra, C.F. de & 1 & 180.372 & 34,7 & 1 & 183.964 & 33,1 & 1 & 195.943 & 30,9 \\
\hline Rioja, La & 1 & 122.254 & 46,4 & 1 & 133.058 & 48,1 & 1 & 152.698 & 47,8 \\
\hline Ceuta & 1 & 67.615 & 100,0 & 1 & 71.505 & 100,0 & 1 & 83.185 & 100,0 \\
\hline Melilla & 1 & 56.600 & 100,0 & 1 & 66.411 & 100,0 & 1 & 80.655 & 100,0 \\
\hline TOTAL & 116 & 20.049 .737 & 51,6 & 139 & 21.550 .443 & 52,8 & 147 & 24.534 .189 & 52,7 \\
\hline 5> POB. EST. 2011 & 68 & 13.808 .042 & 56,7 & 91 & 14.986 .833 & 58,2 & 97 & 17.103.794 & 57,6 \\
\hline
\end{tabular}

Leyenda: N. ${ }^{\circ}$ MUN.: municipios estudiados / POB. EST: población estudiada /\%POB. EST.: porcentaje de población estudiada respecto a la población total en cada fecha (en 2011 población total residente en viviendas principales) / Resaltados los cinco valores mayores en $\mathrm{N}^{\circ} \mathrm{MUN}$. y POB. EST., y los valores superiores al promedio estatal en \%POB. EST. / CC. AA ordenadas por tamaño de población estudiada en 2011.

FIG. 2 / Ámbito de estudio de los Catálogos de 1991, 2001 y 2011.

Fuente: elaboración propia a partir de HERNÁNDEZ \& al. (2018b).

${ }^{4}$ El tamaño y criterios de delimitación de las secciones censales se establece en las instrucciones técnicas a los Ayuntamientos sobre la gestión del Padrón Municipal, cuya última actualización es del año 2020 (MINISTERIO DE LA
Presidencia, Relaciones con las Cortes y Memoria DEMOCRÁtICA, 2020: apartado 14.2). Las secciones censales no pueden superar los 2.500 habitantes o 2.000 electores, y han de tener un mínimo de 500 electores. 
se trata de un perímetro instrumental previo al trabajo de delimitación del BV y del que se obtienen los variables con las que se calculan los indicadores de vulnerabilidad. EI BV es una delimitación urbanística de tejidos con homogeneidad morfotipológica y continuidad espacial (FIG. 3).

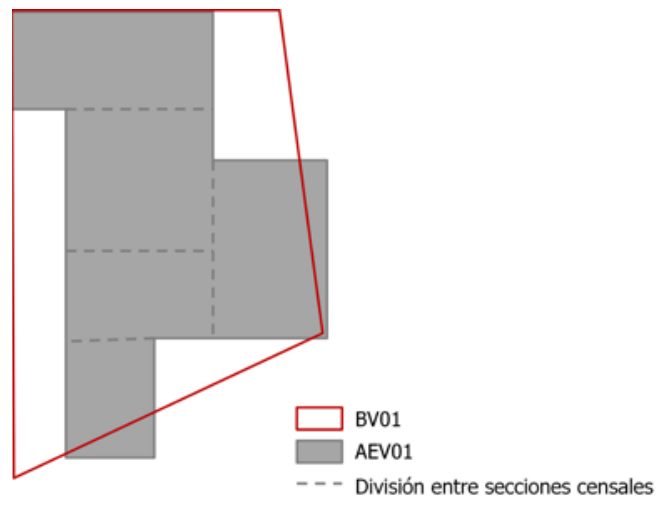

FIG. 3/ Representación esquemática de un Barrio Vulnerable (BV) y un Área Estadística Vulnerable (AEV).

Fuente: HERNÁNDEZ \& al. (2018b).

Las delimitaciones de los Barrios Vulnerables se realizan, mediante un doble proceso de análisis estadístico y urbanístico espacial, siguiendo la siguiente metodología:

- Definición de los Indicadores Básicos de Vulnerabilidad Urbana (IBVU).

- Obtención de las variables desagregadas por sección censal que permiten calcular los IBVU.

- Establecimiento de los Valores de Referencia (VR) para los IBVU que permiten considerar un área como vulnerable.

- Delimitación de Áreas Estadísticas Vulnerables (AEV), mediante el cálculo de los indicadores básicos para agrupaciones de secciones censales que superasen alguno de los valores de referencia. Se realizó en gabinete mediante un proceso de tanteo en el que se incorporaron a las delimitaciones tantas secciones censales como fuera posible, siempre que se encontraran dentro de los límites poblacionales establecidos (3.500 a 15.000 habitantes) y que no

\footnotetext{
${ }^{5}$ La metodología detallada utilizada en el Catálogo de 2011 puede ser consultada en el documento "Síntesis Metodológica general del Catálogo de Barrios Vulnerables e Indicadores
}

presentaran fracturas significativas en los tejidos urbanos incluidos.

- Delimitación de Barrios Vulnerables (BV). Todas las ediciones del Catálogo se han realizado buscando delimitar áreas espaciales con un suficiente grado de homogeneidad y continuidad espacial que permitiese actuar sobre ellas con las herramientas propias del planeamiento urbano. Para ello las AEV se revisaron mediante trabajo de gabinete y de campo, en el que se verificaron y contrastaron con los servicios técnicos municipales, para englobar tejidos sin discontinuidades espaciales significativas, morfológicas o debidas a la estructura de la ciudad, de forma que el resultado final fuese la delimitación de un Barrio Vulnerable, sobre el que se pudiesen desarrollar proyectos de carácter sociourbanístico.

En los Catálogos de 1991 y 2001 se delimitaron ambos perímetros (AEV y BV). Para el año 2011 se inició el estudio a partir de las delimitaciones de barrios vulnerables de 2001, comprobando en primer lugar si era posible establecer un área estadística vulnerable asociada a su perímetro ${ }^{5}$. A continuación, se delimitaron las nuevas áreas estadísticas vulnerables que pudieran existir sin vinculación a los barrios vulnerables delimitados en 2001 y sin llegar a delimitar barrios vulnerables para ellas. De este modo, el Catálogo de 2011 se compone de dos tipos de delimitaciones: barrios vulnerables de 2001 que continuaban siéndolo en 2011, y áreas estadísticas vulnerables nuevas, ajenas a las delimitaciones urbanísticas de 2001, y para las que sólo se dispone de delimitaciones estadísticas.

Las áreas delimitadas en los Catálogos no excluyen la existencia de otras áreas en cada una de las ciudades que no cumplan el tamaño mínimo y las condiciones de homogeneidad y continuidad espacial exigidas.

\subsubsection{Indicadores de vulnerabilidad}

Las delimitaciones de barrios vulnerables se realizaron a partir de tres Indicadores Básicos de Vulnerabilidad Urbana (IBVU), dos de ellos referidos a las condiciones socioeconómicas, nivel de estudios (IEST) y de paro (IPAR), y el tercero a la calidad de la vivienda, determinado a través de las carencias en la edificación (IVIV).

Básicos de Vulnerabilidad Urbana" (MINISTERIO DE FOMENTO, 2016). 
Las dimensiones de la vulnerabilidad analizadas se han mantenido en los tres Catálogos, por la sencillez de sus indicadores, algo que facilita su interpretación y comunicación, y para permitir la comparación temporal. La definición de los indicadores parte de la propuesta realizada en 1996 por la OCDE en la que se planteaban dos indicadores: tasa de paro y nivel de renta. El primero pudo reproducirse al disponer de datos, pero para el segundo no se disponía de información estadística en España, por lo que se decidió utilizar dos variables proxy que mostraban una alta correlación con la renta: el nivel de estudios y las carencias en la vivienda.

Para determinar la vulnerabilidad de un área se establecieron Valores de Referencia (VR) para cada uno de los indicadores y años referidos a los valores estatales para cada indicador. Así, las áreas detectadas como vulnerables son aquellas que presentan peores indicadores que los estatales.

Las modificaciones metodológicas introducidas en cada Censo, especialmente en el de 2011, así como las transformaciones socioeconómicas a lo largo de las dos décadas que abarcan los Catálogos, hicieron necesario revisar los indicadores y valores de referencia utilizados. La definición de indicadores es la siguiente (FIG. 4).

- IEST. Indicador de Estudios. El indicador fue el mismo en los tres años: porcentaje de población analfabeta o sin estudios, aunque con cambios en el universo de cálculo (en 1991 población entre 10 y 64 años, y en 2001 y 2011 la población mayor de 16 años).

- IPAR. Indicador de Paro. El indicador no se modificó en los tres Catálogos: porcentaje de población (de 16 años o más) en situación de paro respecto a la población activa (de 16 años o más).

Los valores de referencia se fijaron en 1,5 veces el indicador estatal para los años
1991 (28\%) y 2001 (21,30\%). En 2011 el nivel de paro como consecuencia de la crisis era muy elevado y el indicador estatal era del $29,64 \%$, por lo que establecer el valor de referencia en 1,5 veces conllevaría infrarrepresentar la vulnerabilidad por este indicador. Para solventar este problema se estableció el valor de referencia en aquel nivel del indicador que detectase un volumen de secciones censales vulnerables equivalente al detectado en 2001 , fijándose en el $42,33 \%$.

- IVIV. Indicador de Vivienda. El indicador utilizado fue distinto en cada uno de los años.

- 1991. Se utilizaron tres indicadores: porcentaje de viviendas principales sin agua corriente (IVIV1), sin retrete o aseo (IVIV2), y sin baño o ducha (IVIV3). Los valores de referencia se fijaron en el 2,3 y $5 \%$ respectivamente. Se consideraron vulnerables las áreas que superasen el valor de referencia en cualquier de ellos.

- 2001. Las variables utilizadas en 1991 no existieron en el Censo de 2001. Se utilizó como nuevo indicador el porcentaje de población residente en viviendas principales sin aseo y/o ducha. El valor de referencia se fijó el $2 \%$, el doble del indicador estatal.

- 2011. Los cambios metodológicos en el Censo de 2011, en el que los resultados se infieren de una muestra, impiden disponer de información coherente desagregada espacialmente para variables en las que la categoría buscada se refiera a grupos pequeños, como era el caso del indicador de vivienda utilizado en 2001, cuyo indicador estatal era ese año de tan sólo el 1\%. El indicador propuesto fue el porcentaje de vivienda familiares situadas en edificios cuyo

\begin{tabular}{|c|c|c|c|c|c|c|c|}
\hline & & \multicolumn{2}{|c|}{1991} & \multicolumn{2}{|c|}{2001} & \multicolumn{2}{|c|}{2011} \\
\hline & & IBVU & VR & IBVU & VR & IBVU & VR \\
\hline IEST. Estudios & & $18,78 \%$ & $>28 \%$ & $15,30 \%$ & $>22,95 \%$ & $10,92 \%$ & $>16,38 \%$ \\
\hline IPAR. Paro & & $19,30 \%$ & $>28 \%$ & $14,20 \%$ & $>21,30 \%$ & $29,64 \%$ & $>42,33 \%$ \\
\hline \multirow{3}{*}{ IVIV. Vivienda } & IVIV 1 & $0,66 \%$ & $>2 \%$ & \multirow{3}{*}{$1,00 \%$} & \multirow{3}{*}{$>2,00 \%$} & \multirow{3}{*}{$6,99 \%$} & \multirow{3}{*}{$>17,50 \%$} \\
\hline & IVIV 2 & $2,84 \%$ & $>3 \%$ & & & & \\
\hline & IVIV 3 & $4,51 \%$ & $>5 \%$ & & & & \\
\hline
\end{tabular}

FIG. 4 / Indicadores Básicos de Vulnerabilidad Urbana (IBVU) estatales y Valores de Referencia (VR). 
estado de conservación es malo, ruinoso o deficiente. El valor de referencia se estableció en un $17,5 \%$, con el que se detectaba un volumen equivalente de secciones censales al detectado en 2001. Se comprobó que existía una alta correspondencia entre los patrones de distribución espacial de las secciones censales detectadas en 2001 y 2011, lo que permitiría la comparación con los Catálogos anteriores. El nuevo indicador utilizado resultaba, además, más coherente con los problemas del parque de viviendas y las políticas desarrolladas por las distintas administraciones.

\subsubsection{Las concentraciones de población inmigrante}

Junto al Catálogo de 2001 se elaboró una Adenda en la que se estudiaban las concentraciones de población extranjera en las mismas ciudades. Este mismo análisis se repitió en el Catálogo de 2011. El objetivo no era añadir un cuarto indicador para la detección de población vulnerable, identificando población extranjera con vulnerabilidad, sino estudiar las interferencias espaciales entre ambos fenómenos.

El indicador utilizado en 2006 fue el porcentaje de población extranjera procedente de países no pertenecientes a la UE-15 respecto a la población total. Los datos para la Adenda de 2006 se obtuvieron del Padrón de 2006 y el valor del indicador estatal ese año era del $8,77 \%$ para las ciudades mayores de 50 mil habitantes. En 2011 el indicador fue el porcentaje de población nacida en países no pertenecientes a la UE- 15 respecto a la población total, la fuente de datos fue el Censo 2011, y el valor estatal era el 10,34\%. El valor de referencia se fijó para ambos años en el $20 \%$, permitiendo comparar la evolución real en el periodo.

\subsubsection{Sistema de clasificación}

Los catálogos contemplan dos de los aspectos a los que hace referencia el empleo del concepto de vulnerabilidad: la diversidad de dimensiones implicadas, y sus diferentes niveles de intensidad.

El carácter multidimensional se recoge con la utilización de tres indicadores, referidos dos de ellos a las condiciones socioeconómicas de la población y el otro a las del soporte residencial. En cada área delimitada pueden darse una o más de estas dimensiones de la vulnerabilidad.

Los niveles de intensidad se evalúan mediante un sistema de clasificación que permite graduar el amplio espectro de situaciones que pueden ir desde las más leves, en posiciones de riesgo previo a que se puedan producir consecuencias reales para sus habitantes, a las más agudas, en las que la población se encuentra ya en una situación de exclusión social.

El sistema de clasificación propuesto pretende ser un método sencillo que permita representar y comunicar el nivel de vulnerabilidad en cada una de las áreas delimitadas.

Para ello, en primer lugar, se caracterizan los tres indicadores básicos de cada área en función de su cociente con el valor del indicador estatal. Para los indicadores de paro y estudios se establecen 4 niveles y para el de vivienda 5 , según la escala: $0, A, B, C \circ D$ (FIG. 5), en función del número de veces que superan el valor estatal de cada indicador. Los valores utilizados para cada uno de los niveles, indicadores y años pueden ser consultados en HERNÁNDEZ \& al. (2018b).

Una vez establecidos los niveles para cada indicador básico se realiza la clasificación en dos fases utilizando matrices de doble entrada. En la primera se clasifica el área en función de su nivel de vulnerabilidad socioeconómica, mediante la intersección de los niveles de los dos

\begin{tabular}{|c|c|c|}
\hline \multirow{2}{*}{$\begin{array}{c}\text { Nivel de } \\
\text { Vulnerabilidad }\end{array}$} & \multicolumn{2}{|c|}{ Cociente entre el indicador básico de vulnerabilidad y la media nacional } \\
\hline & Criterios IEST y IPAR & Criterios IVIV \\
\hline 0 & $<1$ & $<1$ \\
\hline A & $\geq 1$ y $<$ VR. Valor de Referencia & $\geq 1 \mathrm{y}<$ VR. Valor de Referencia \\
\hline B & $\geq$ VR. Valor de Referencia y $<2,5$ & $\geq$ VR. Valor de referencia $y<4$ \\
\hline $\mathrm{C}$ & $\geq 2,5$ & $\geq 4 y<8$ \\
\hline $\mathrm{D}$ & & $\geq 8$ \\
\hline
\end{tabular}

FIG. 5 / Criterios para los niveles de vulnerabilidad de los indicadores básicos de vulnerabilidad. 


\begin{tabular}{|c|c|c|c|c|c|c|}
\hline & & & \multicolumn{4}{|c|}{ PARO -Niveles IPAR- } \\
\hline & & & 0 & A & B & C \\
\hline & & & 0-MN & MN-VR & VR-2,5MN & $>2,5 M$ \\
\hline \multirow{4}{*}{$\begin{array}{c}\text { ESTUDIOS } \\
\text {-Niveles IEST- }\end{array}$} & 0 & $0-\mathrm{MN}$ & NV & NV & VL & VS \\
\hline & A & MN-VR & NV & NV & $\mathbf{v}$ & VS \\
\hline & $\mathrm{B}$ & VR-2,5MN & VL & $\mathbf{v}$ & $\mathbf{V}$ & VS \\
\hline & $\mathrm{C}$ & $>2,5 \mathrm{MN}$ & VS & VS & VS & VC \\
\hline
\end{tabular}

Leyenda: MN: media nacional / VR: valor de referencia / Grados de vulnerabilidad: VC: crítica, VS: severa, V: media, VL: leve.

FIG. 6/ Clasificación de los barrios vulnerables según su grado de vulnerabilidad socioeconómica.

Fuente: HERNÁNDEZ \& al. (2018b).

\begin{tabular}{|c|c|c|c|c|c|c|}
\hline & & \multicolumn{5}{|c|}{ VIVIENDA - Niveles IVIV- } \\
\hline & & $\mathbf{0}$ & A & B & C & D \\
\hline & & $0-M N$ & MN-VR & VR-4MN & $4 M N-8 M N$ & $18 \mathrm{MN}$ \\
\hline \multirow{5}{*}{$\begin{array}{l}\text { Clasificación } \\
\text { ESTUDIOS } \\
\text { + PARO }\end{array}$} & NV. No vulnerable & NV & NV & VL & VL & V \\
\hline & VL. Vulnerabilidad leve & VL & VL & VL & $\mathbf{v}$ & VS \\
\hline & V. Vulnerabilidad media & V & $\mathbf{v}$ & $\mathbf{v}$ & VS & VC \\
\hline & VS. Vulnerabilidad severa & VS & VS & VS & VC & VC \\
\hline & VC. Vulnerabilidad crítica & vc & vc & VC & vc & VC \\
\hline
\end{tabular}

Leyenda: MN: media nacional / VR: valor de referencia / Grados de vulnerabilidad: VC: crítica, VS: severa, V: media, VL: leve.

FIG. 7 / Clasificación global de los barrios vulnerables según su grado de vulnerabilidad.

Fuente: HERNÁNDEZ \& al. (2018b).

indicadores referidos a estas dimensiones, estudios y paro (FIG. 6).

Esta clasificación de carácter sociodemográfico nos permite caracterizar las poblaciones que habitan en cada uno de los barrios delimitados en función de su capacidad para afrontar una posible crisis, a través de su relación con el empleo y la formación, asociadas a la integración económica y a la relacional; pero el Catálogo busca ampliar estás dimensiones con la del espacio en que se habita, de forma que las características de las viviendas en las que se reside pueden suponer un incremento de la vulnerabilidad de unas poblaciones frente a otras mejor alojadas. Para ello, el nivel de vulnerabilidad socioeconómica de la población que habita en el barrio (o área estadística) se cruza con el nivel de vulnerabilidad residencial, considerado como una dimensión que podría empeorar el nivel de vulnerabilidad de la población que allí habita, obteniendo de esta manera la clasificación global del grado de vulnerabilidad del área (FIG. 7). La clasificación de vulnerabilidad global se entiende, por tanto, como la intersección de la vulnerabilidad socioeconómica, entendida

${ }^{6}$ Aunque en el Catálogo Urbanístico de Barrios Vulnerables de 2001 fueron incorporados oficialmente 624 barrios, tres de ellos no superaban los valores de referencia establecidos: Los como vulnerabilidad estructural, con la residencial, entendida como vulnerabilidad contextual, de forma que esta última puede contribuir a incrementar la vulnerabilidad de la población en función de la calidad de la vivienda en que habita. El sistema contempla cuatro grados de Vulnerabilidad: Leve (VL), Media (V), Severa (VS), y Crítica (VC).

\subsection{Principales resultados}

\subsubsection{Barrios y población total vulnerable}

El ámbito de los Catálogos fue de 116 ciudades en 1991, 139 en 2001 y 147 en 2011 . Se detectaron 370 barrios vulnerables en 81 ciudades en $1991,621^{6}$ barrios vulnerables en 103 ciudades en 2001, y 918 barrios y áreas estadísticas vulnerables en 132 ciudades en 2011. Si bien los Catálogos incrementaron su ámbito de estudio en las tres fechas analizadas, se incrementó aún más la población que habitaba en

Mallos Sur (A Coruña), Poblado de Absorción de Hortaleza (Madrid) y San José (Murcia). 
ámbitos vulnerables y su concentración espacial. La población analizada aumentó en algo más del $22 \%$ entre 1991 y 2011 y la población en ámbitos vulnerables en más del $136 \%$. En número de barrios vulnerables el incremento fue aún más significativo, pasando de 370 en 1991 a 918 en 2011. En 2011 habitaban en barrios vulnerables casi 6,7 millones de personas, un $27 \%$ de la población analizada (FIG. 8), frente a los 2,8 millones y el $14 \%$ en 1991.

En las tablas no se incluyen la población vulnerable que reside en secciones censales que aun superando los valores de referencia para los IBVU no forman parte de un Barrio Vulnerable o de un Área Estadística Vulnerable. Reconociendo la necesidad de intervenir también sobre ámbitos menores de población vulnerable distribuida de forma dispersa, no forma parte del objetivo de los Catálogos, que es el de delimitar barrios sobre los que se pueda actuar mediante planes y proyectos de carácter urbanístico. En todo caso, se realizó el cálculo de la población incluida en secciones censales no contenida en los barrios delimitados, comprobándose que la población vulnerable se localiza mayoritariamente concentrada en barrios vulnerables, con tan solo un $9 \%$ de la población vulnerable en 2011 alojada en secciones censales exteriores a ellos.

En cualquier caso, tanto por el incremento del número de barrios y áreas estadísticas delimitadas, como por el incremento de la población que habita en ellos, podemos concluir que, en el periodo considerado en los Catálogos, los desequilibrios internos de las ciudades españolas aumentaron de forma significativa y continuada, independientemente de las coyunturas económicas y políticas.

\subsubsection{Distribución territorial de la vulnerabilidad}

La distribución territorial de la vulnerabilidad ha variado poco en los tres Catálogos. Cinco comunidades autónomas concentran más del $80 \%$ de los barrios y de la población vulnerable en los tres Catálogos. Ordenadas por población vulnerable, son: Andalucía, Cataluña, Comunidad de Madrid, Comunitat Valenciana, y Canarias (FIG. 9). Los mayores incrementos producidos entre 1991 y 2011 se dieron también en estas comunidades, en las que se localizaban 457 de los 548 barrios que incorporó el Catálogo después de su primera edición sobre un total de 918 en 2011. Las comunidades que más barrios incorporaron entre 1991 y 2011 fueron: Cataluña, con 147 de 188; Madrid, con 116 de 140; Comunidad Valenciana, con 104 de 128; Andalucía, con 55 de 215; y Canarias, con 35 de 69.

Las ciudades en las que se detectaron barrios vulnerables fueron ampliándose en cada Catálogo. En 1991 no se detectaron barrios en 35 ciudades, sobre un total de 116; en 2001 en 36 sobre 139; y en 2011 en sólo 15 ciudades de las 147 analizadas no se catalogaron barrios o áreas estadísticas vulnerables. El porcentaje de la población estudiada que reside en barrios vulnerables ha empeorado de forma continuada durante todo el periodo, pasando del $14,1 \%$ en 1991 , al $20,6 \%$ en 2001 y al $27,3 \%$ en 2011 . En las cinco comunidades autónomas con mayor población vulnerable la población incluida en las áreas estudiadas alcanzó el 32,7\% en 2011.

La distribución territorial de la vulnerabilidad se ajusta a los patrones conocidos de desigualdad socioeconómica en la geografía española. La

\begin{tabular}{|c|c|c|c|c|c|c|}
\hline & \multirow{2}{*}{1991} & \multirow{2}{*}{2001} & \multirow{2}{*}{2011} & \multicolumn{3}{|c|}{ Incremento } \\
\hline & & & & 91-01 & $01-11$ & 91-11 \\
\hline Población total & 38.872 .268 & 40.847 .371 & 46.815 .916 & $5,1 \%$ & $14,6 \%$ & $20,4 \%$ \\
\hline Población analizada & 20.049 .737 & 21.550 .443 & 24.534 .189 & $7,5 \%$ & $13,8 \%$ & $22,4 \%$ \\
\hline Población en BBVV & 2.832 .080 & 4.441 .041 & 6.697 .400 & $56,8 \%$ & $50,8 \%$ & $136,5 \%$ \\
\hline N. ${ }^{\circ}$ de ciudades estudiadas & 116 & 139 & 147 & $19,8 \%$ & $5,8 \%$ & $26,7 \%$ \\
\hline N..$^{\circ}$ de ciudades con BBVV & 81 & 103 & 132 & $27,2 \%$ & $28,2 \%$ & $63,0 \%$ \\
\hline N. ${ }^{\circ} \mathrm{BBVV}$ & 370 & 621 & 918 & $67,8 \%$ & $47,8 \%$ & $148,1 \%$ \\
\hline
\end{tabular}

Leyenda: "Población total" según los censos de 1991, 2001 y 2011 / "Población analizada" total de las ciudades estudiadas / "Población en BBVV" que habitaba en los barrios vulnerables detectados en las ciudades estudiadas / " $N .^{\circ}$ de ciudades estudiadas": ciudades mayores de 50.000 habitantes y capitales de provincia (en 2011 están incluidas aquellas ciudades menores de 50.000 habitantes que si que los superaban en 2001) / "N. 'de ciudades con BBVV": ciudades en las que se detectaron barrios vulnerables / "N. ${ }^{\circ}$ de BBVV": número de barrios vulnerables detectados (en 2011 barrios vulnerables y áreas estadísticas vulnerables). 


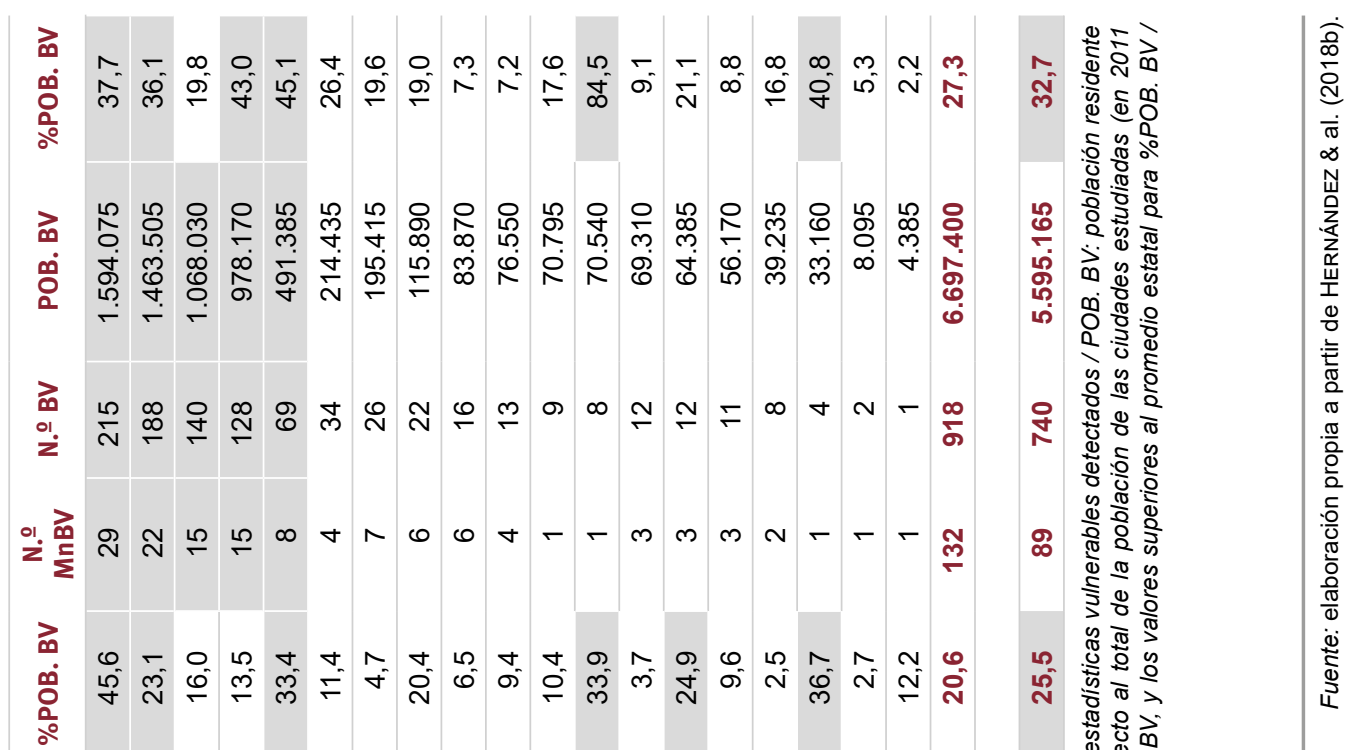

ว. 䒘

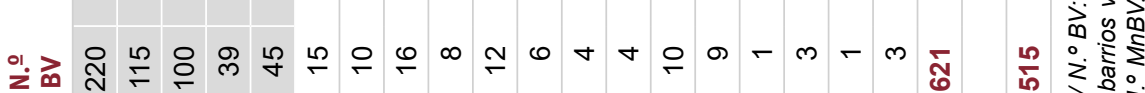
i.

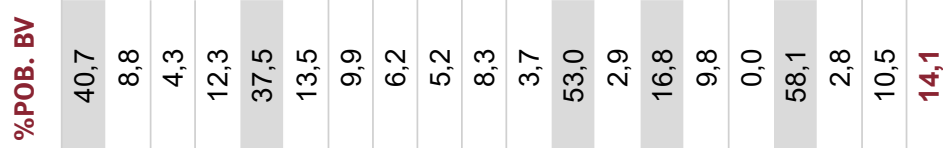

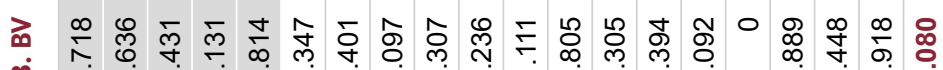
音

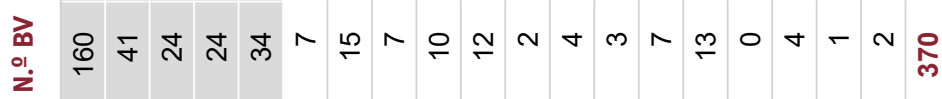

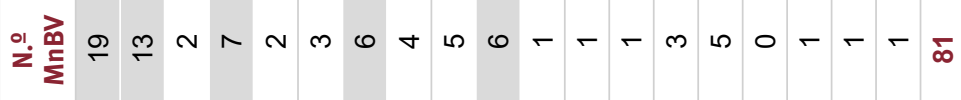

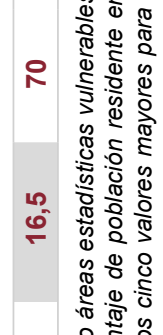
ํ.

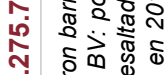
A

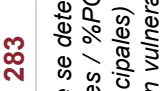

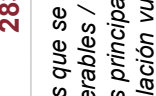

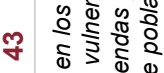
का

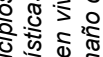

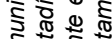

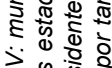

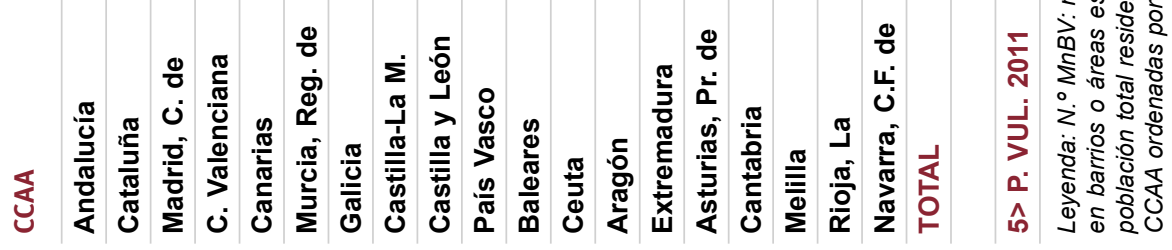


mitad norte del país concentra los menores índices de vulnerabilidad, alojando a menos del $10 \%$ de su población urbana en los barrios y áreas delimitadas (es el caso de Asturias, País Vasco, Navarra, La Rioja, Asturias y Aragón), mientras que los mayores índices se dan en la ciudad autónoma de Ceuta y en Canarias. Andalucía mejoró entre 1991 y 2011, aunque aún presentaba en 2011 uno de los mayores índices del estado. Cataluña, Comunidad de Madrid y Comunidad Valenciana fueron las comunidades en las que más empeoró la vulnerabilidad, y de ellas sólo Madrid se encontraba por debajo de la media estatal en 2011.

\subsubsection{Tipos de vulnerabilidad}

Por tipo de vulnerabilidad (FIG. 10), el grupo más numeroso de barrios fue detectado por el indicador de paro en 1991, por los de estudios y/o paro en 2001, y por el de estudios en 2011. La diferencia más apreciable respecto a los tipos de vulnerabilidad se dio en el Catálogo de 1991, en el que más un $75 \%$ de la población vulnerable fue detectada por el indicador de paro.
En los dos Catálogos posteriores los tipos de vulnerabilidad se equilibraron de forma notable y ninguno de los tipos de vulnerabilidad afectaba a más de la mitad de los barrios.

La mayoría de las áreas delimitadas como vulnerables lo eran respecto a un único indicador, aunque más del $40 \%$ de ellas en 1991, el $30 \%$ en 2001 y el $17,1 \%$ en 2011 presentaban más de una de las dimensiones de la vulnerabilidad (FIG. 11). En consecuencia, el incremento de barrios catalogados entre 1991 y 2011 se produjo en barrios vulnerables por un solo indicador.

\subsubsection{Niveles de vulnerabilidad}

La mayor parte de los Barrios Vulnerables incluidos en los catálogos de 1991, 2001 y 2011 presentaban niveles de vulnerabilidad moderados (leve o media). La población vulnerable que habitaba en barrios con niveles de vulnerabilidad altos (crítica y severa) se redujo del $11,7 \%$ en 1991, al 8,4\% en 2001 y al $4,5 \%$ en 2011 (FIG. 12). Por tanto, los incrementos de población vulnerable que se produjeron en los Catálogos de

\begin{tabular}{|c|c|c|c|c|c|c|c|c|c|}
\hline & \multicolumn{3}{|c|}{1991} & \multicolumn{3}{|c|}{2001} & \multicolumn{3}{|c|}{2011} \\
\hline & $\begin{array}{l}\text { N. } .9 \\
\text { BBVV }\end{array}$ & POB. BBVV & $\begin{array}{c}\text { \%POB. } \\
\text { BBVV }\end{array}$ & $\begin{array}{c}\text { N. } .9 \\
\text { BBVV }\end{array}$ & POB. BBVV & $\begin{array}{c}\text { \%POB. } \\
\text { BBVV }\end{array}$ & $\begin{array}{l}\text { N. } .9 \\
\text { BBVV }\end{array}$ & $\begin{array}{l}\text { POB. } \\
\text { BBVV }\end{array}$ & $\begin{array}{c}\text { \%POB. } \\
\text { BBVV }\end{array}$ \\
\hline IEST. Estudios & 133 & 992.346 & 35,0 & 287 & 2.014 .769 & 45,4 & 403 & 2.943 .605 & 44,0 \\
\hline IPAR. Paro & 274 & 2.147 .412 & 75,8 & 284 & 2.140 .047 & 48,2 & 318 & 2.229 .180 & 33,3 \\
\hline IVIV. Vivienda & 167 & 1.205 .393 & 42,6 & 266 & 1.835 .457 & 41,3 & 387 & 2.911 .255 & 43,5 \\
\hline TOTAL & 370 & 2.832 .080 & 100,0 & 621 & 4.441 .041 & 100,0 & 918 & 6.697 .400 & 100,0 \\
\hline
\end{tabular}

Leyenda: $N .^{\circ} B B V V$ : barrios o áreas estadísticas vulnerables detectados / POB. BBVV: población residente en barrios o áreas estadísticas vulnerables /\%POB. BBVV.: porcentaje de población vulnerable por cada indicador respecto al total de la población vulnerable / Resaltados los grupos mayores de población y barrios detectados en cada año.

FIG. 10/ Población en barrios vulnerables por indicador en 1991, 2001 y 2011.

Fuente: elaboración propia a partir de HERNÁNDEZ \& al. (2018b).

\begin{tabular}{|c|c|c|c|c|c|c|}
\hline & \multicolumn{2}{|c|}{1991} & \multicolumn{2}{|c|}{2001} & \multicolumn{2}{|c|}{2011} \\
\hline & N.ํ BBVV & $\%$ BBVV & $\begin{array}{c}\text { N.. } \\
\text { BBVV }\end{array}$ & $\%$ BBVV & $\begin{array}{c}\text { N. } .9 \\
\text { BBVV }\end{array}$ & $\%$ BBVV \\
\hline Vulnerables en sólo 1 dimensión & 209 & 56,5 & 436 & 70,2 & 761 & 82,9 \\
\hline Vulnerables en 2 dimensiones & 118 & 31,9 & 154 & 24,8 & 124 & 13,5 \\
\hline Vulnerables en las 3 dimensiones & 43 & 11,6 & 31 & 5,0 & 33 & 3,6 \\
\hline TOTAL & 370 & 100,0 & 621 & 100,0 & 918 & 100,0 \\
\hline
\end{tabular}

FIG. 11/ Polivulnerabilidad en los barrios vulnerables delimitados en 1991, 2001 y 2011. 


\begin{tabular}{|c|c|c|c|c|c|c|c|c|c|}
\hline \multirow{2}{*}{$\begin{array}{l}\text { Niveles de } \\
\text { vulnerabilidad }\end{array}$} & \multicolumn{3}{|c|}{1991} & \multicolumn{3}{|c|}{2001} & \multicolumn{3}{|c|}{2011} \\
\hline & РОВ. & $\begin{array}{l}\text { N. } .9 \\
\text { BBVV }\end{array}$ & $\begin{array}{l}\text { \%POB. } \\
\text { BBVV }\end{array}$ & POB. & $\begin{array}{l}\text { N.. } .9 \\
\text { BBVV }\end{array}$ & $\begin{array}{l}\text { \%POB. } \\
\text { BBVV }\end{array}$ & POB. & $\begin{array}{l}\text { N.. } . \\
\text { BBVV }\end{array}$ & $\begin{array}{l}\text { \%POB. } \\
\text { BBVV }\end{array}$ \\
\hline VC. Crítica & 117.267 & 16 & 4,1 & 77.461 & 13 & 1,7 & 62.135 & 10 & 0,9 \\
\hline VS. Severa & 213.774 & 29 & 7,5 & 295.952 & 36 & 6,7 & 241.505 & 33 & 3,6 \\
\hline V. Media & 1.598 .072 & 203 & 56,4 & 2.058 .868 & 285 & 46,4 & 2.873 .265 & 392 & 42,9 \\
\hline VL. Leve & 902.967 & 122 & 31,9 & 2.008 .760 & 287 & 45,2 & 3.520 .495 & 483 & 52,6 \\
\hline TOTAL & 2.832 .080 & 370 & 100,0 & 4.441 .041 & 621 & 100,0 & 6.697 .400 & 918 & 100,0 \\
\hline VC+VS & 331.041 & 45 & 11,7 & 373.413 & 49 & 8,4 & 303.640 & 43 & 4,5 \\
\hline
\end{tabular}

FIG. 12/ Niveles de vulnerabilidad de los barrios y áreas estadísticas vulnerables en 1991, 2001 y 2011.

2001 y 2011 fueron en barrios con vulnerabilidades moderadas (leve o media).

\subsubsection{Concentraciones de población inmigrante y vulnerabilidad}

La población inmigrante se concentró espacialmente de forma muy acusada en el periodo analizado entre 2006 y 2011. La población que vivía en barrios con concentraciones significativas de población inmigrante (más del $20 \%$ ) era de poco más de dos millones en 2006 y superaba los 5,3 millones en 2011 , un incremento de casi el $162 \%$, frente al $4,9 \%$ de la población analizada. Las ciudades en las que se detectaron barrios que superan el valor de referencia en inmigración $(20 \%)$ pasaron de 59 a 109 y los barrios de 256 a 697 . Este proceso de concentración de la población inmigrante se produjo con una distribución territorial muy desequilibrada, con predominio en las ciudades del arco mediterráneo y de la Comunidad de Madrid.

Los resultados permiten constatar que vulnerabilidad y concentraciones de población extranjera no siempre son realidades coincidentes. Aunque un número importante de los barrios vulnerables en las grandes ciudades coinciden con los delimitados por población inmigrante; sin embargo, existen barrios con altas concentraciones de inmigrantes que no aparecen como vulnerables. Esta situación abre nuevas perspectivas de análisis sobre la distribución espacial y la capacidad de integración de la población de origen inmigrante, puesto que estas poblaciones se encuentran en sus primeras etapas de integración y tienden a alojarse en espacios con precios inmobiliarios bajos, coincidentes con aquellos que podrían considerase como vulnerables o sobre los que pueda ser necesario intervenir para evitar que en el futuro lo sean.

\subsection{Distribución de la información y formatos disponibles}

\subsubsection{Productos elaborados}

El principal producto de los Catálogos es la delimitación y caracterización de los barrios vulnerables, sobre los que sería posible la ejecución de intervenciones de rehabilitación urbana integrada. Estos barrios se basan en la delimitación de áreas estadísticas vulnerables, que sirven de base para su perimetrización como posibles áreas finalistas de planes y programas de actuación sociourbanística. A los barrios y áreas estadísticas se suman las bases de datos de indicadores asociadas a cada uno de ellos. Para cada una de las áreas o barrios se dispone de sus indicadores básicos de vulnerabilidad, las variables que permiten su cálculo y una batería de variables e indicadores complementarios que permiten caracterizar el área en las dimensiones socioeconómica y residencial.

Los Catálogos incluyen un fichero de municipios con barrios vulnerables y las fichas de éstos, con los siguientes contenidos:

- Ficha de caracterización municipal para cada uno de los municipios en los que existan barrios vulnerables. Existente sólo para los Catálogos de 1991 y 2001.

- Plano de localización de los barrios vulnerables detectados en cada municipio.

- Ficha estadística del barrio o área estadística vulnerable. Con un plano de localización y delimitación, los indicadores básicos y la batería de indicadores complementarios.

- Ficha urbanística del barrio, con un análisis cualitativo del barrio en el que se describen su forma de crecimiento, origen, principales actuaciones públicas desarrolladas, y problemas y oportunidades detectados. Esta ficha 
sólo está disponible para los Catálogos de 1991 y 2001.

Como producto adicional se elaboraron informes generales sobre la vulnerabilidad urbana en España en los tres años y específicos para cada comunidad autónoma en el Catálogo de 2001. Tanto los informes como los ficheros se distribuyen en formato $p d f$.

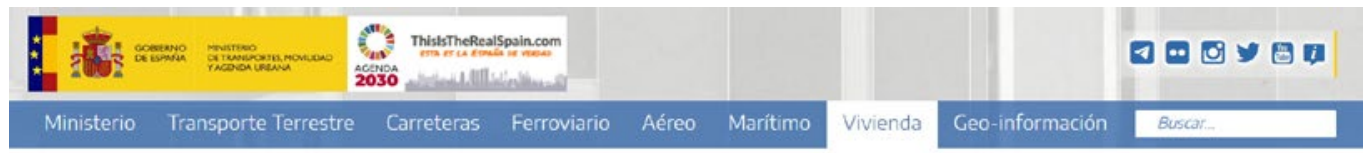

A Vivienda Actividades y servicios > Observatorios

MEDIDAS COVID-19

ALOUILA BIEN, ;ES TU DERECHO!

PLAN ESTATAL DE VIVIENDA 2018-2021

ARQUITECTURA Y EDIFICACION

UREANISMO Y SUELO

| ACTIVIDADES Y SERVICIOS

Organización $y$ funciones

Normativa

Observatorios

Sedes electrónica

Contacto

Estudios y Publicaciones

\section{Visor del Catálogo de Barrios Vulnerables}

Esto Visor permito accoder al Cotalogo do Barrios Vulnorablos, visualizéndalos sobre un mape o sobre una fotografia adrea, y descarger

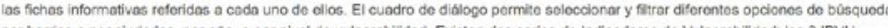

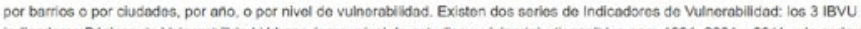
indicadoros Básioos do Vüneroblidad Urbana (Garo, nivel de estudios y vivienda), disponiolos para 1991, 2001 y 2011, y la sorio dol Indicador de Inmigración, disponible para 2006 y 2011. Debajo del mapa se cfroce una Tabla do Resultados con la información corrospondiente de los elomontos seloccionados y visualizados en el mape, que puede tombien ser descergodo pare su consulto.

La información sobre la metodologia de celmitación de los banios y la información sobre los indicadores empleados puede consultarse en el siguiente enlace:

Sintesis Metodologica general del Catdiogo de Barrios Vulnerabies e Indicadores Básicos do Vulnerabilidad Urbana BUSSQUEDA
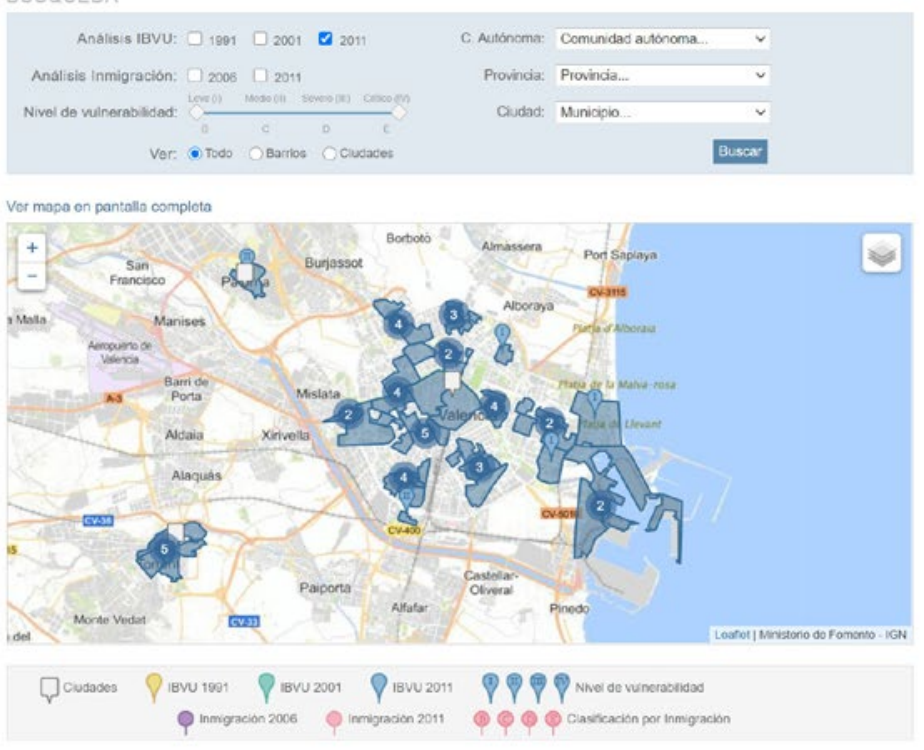

TABLA DE RESULTADOS

\begin{tabular}{|c|c|c|c|c|c|c|}
\hline \multirow[b]{2}{*}{ - Año } & \multirow[b]{2}{*}{ Provincia } & \multirow[b]{2}{*}{ Cludad } & & \multirow[b]{2}{*}{ Barrio } & \multirow{2}{*}{ Exporiar Eucel } & \multirow{2}{*}{$\begin{array}{c}\text { Exportar POF } \\
\text { Nivel }\end{array}$} \\
\hline & & & & & & \\
\hline 2011 & Alava & Vhoria-Casteiz & $\Leftrightarrow$ & 0105901 & [D & Bajo \\
\hline 2011 & Alava & Vhoria-Gastoiz & Es & 0105902 & B & Bajo \\
\hline 2011 & Alava & Vhona-Gastez & 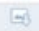 & 0105903 & 18 & $B a j o$ \\
\hline 2011 & Álava & Vacra-Gastez & 40 & Cascos Histínico & (5) & Bajo \\
\hline 2011 & Albacoto & Abacote & {$[4$} & 0200301 & (B) & Bajo \\
\hline 2011 & Albacute & Abacote & $\Leftrightarrow$ & 0200302 & B & Bajo \\
\hline 2011 & Albacete & Ablacule & E) & 0200303 & {$[8$} & Bajo \\
\hline 2011 & Albacote & Abacete & $E$ & Cologio Santo Ángel & BD & Medio \\
\hline 2011 & Albacote & A hacete & 80 & La estrola-la milogrosa & (B) & $\mathrm{Bajo}$ \\
\hline 2011 & Alicanta/Alacant & Alcoy/ANoOi & $\Leftrightarrow$ & 0300901 & CD & Modio \\
\hline
\end{tabular}

FIG. 13/ Visor del Catálogo de Barrios Vulnerables.

Fuente: página electrónica del MINISTERIO DE TRANSPORTES, MOVILIDAD Y AGENDA URBANA. https://portalweb.mitma.es/aplicaciones/portalweb/BarriosVulnerables 


\subsubsection{Herramientas de difusión}

Los resultados de los Catálogos se difunden a través de diversos recursos electrónicos, el principal de los cuales es el portal electrónico del Observatorio de la Vulnerabilidad Urbana $(\mathrm{OVU})^{7}$ del Ministerio de Transportes, Movilidad y Agenda Urbana.

El OVU dispone de un Visor del Catálogo de Barrios Vulnerables en el que es posible visualizar sobre mapa las delimitaciones de los tres Catálogos y filtrar los resultados por fecha, unidades territoriales, o niveles de vulnerabilidad (FIG. 13). Para cada una de las ciudades se puede descargar un mapa de localización de todos los barrios vulnerables (y áreas estadísticas en 2011) delimitados en cada fecha, así como la ficha estadística detallada para cada uno de ellos. El Visor dispone también de un resumen de la metodología utilizada en los tres Catálogos (MINISTERIO DE FOMENTO, 2016).

Los ficheros por municipios y barrios, así como los informes generales de los catálogos de 1991 y 2001 se encuentran disponibles tanto en la página del OVU, como en la de la Biblioteca Ciudades por un Futuro más Sostenible $(\mathrm{CF}+\mathrm{S})^{8}$.

Como productos derivados del Catálogo de Barrios Vulnerables se elaboraron tres publicaciones con formato de atlas en versión papel y disponibles en versión electrónica en el Archivo Digital de la Universidad Politécnica de Madrid (HERNÁNDEZ \& al., 2015, 2018b y 2018c). En ellos se analiza la evolución de la vulnerabilidad urbana en las ciudades mayores de 300 mil habitantes junto con cartografía de detalle para cada una las ciudades, indicadores y fechas de referencia (FIG. 14).

\section{1 | barrios vulnerables e inmigración 13 CIUDADES | Madrid}

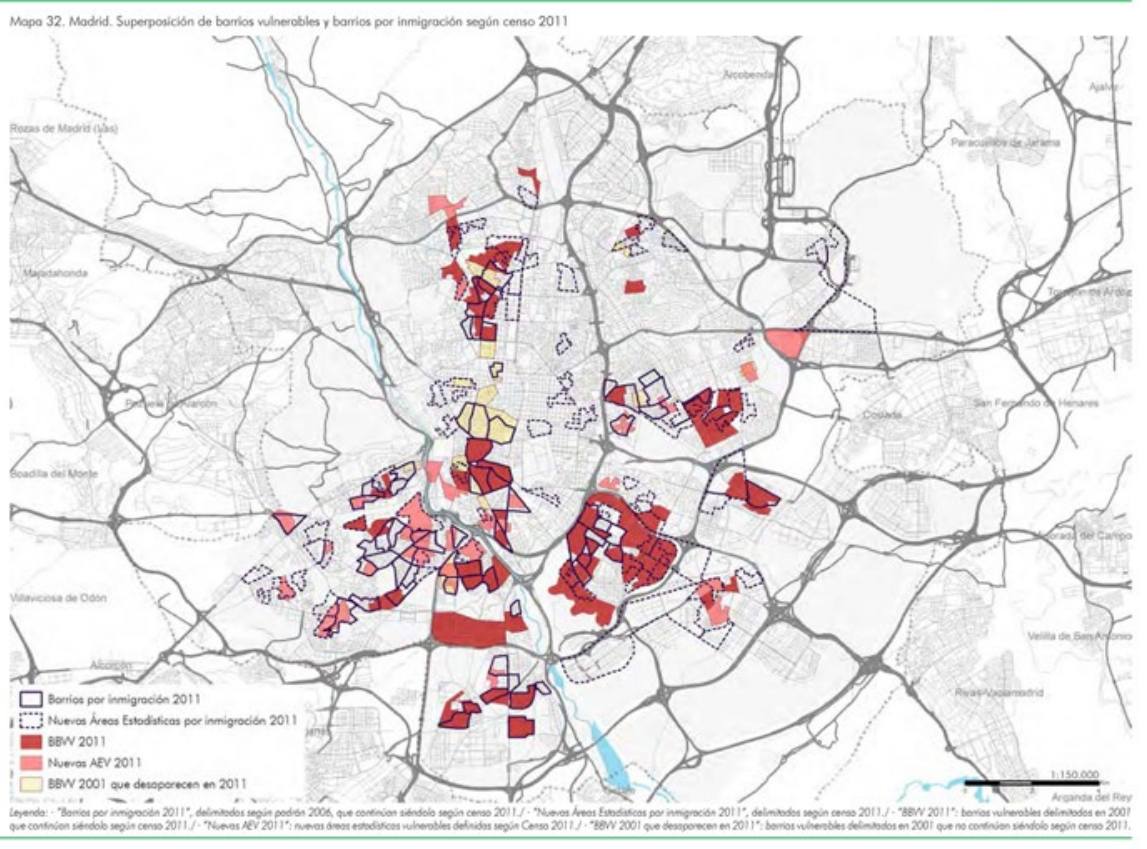

BARRIOS VULNERABLES DE LAS GRANDES CIUDADES ESPAN̂OLAS 13 Ciudades - 1991/2001/2011

Instituto Juan de Herrero - Departamento de Urbanistica y Ordenoción del Territorio - Universidad Politécrica de Madrid

FIG. 14/ Barrios vulnerables y barrios con concentraciones de población inmigrante en la ciudad de Madrid en 2011.

Fuente: HERNÁNDEZ \& al. (2018b).

${ }^{7}$ https://www.mitma.gob.es/arquitectura-vivienda-y-suelo/ur-

${ }^{8}$ http://habitat.aq.upm.es/bbvv/ dad-urbana 


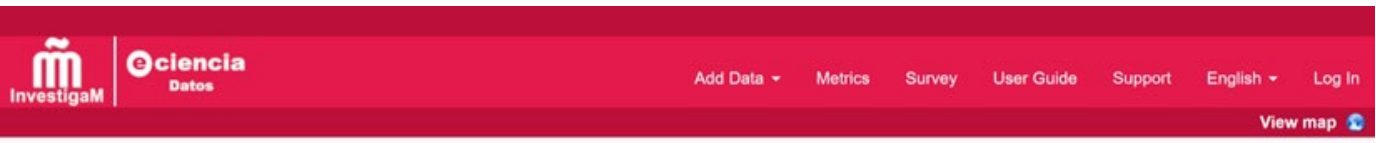

Catálogos de Barrios Vulnerables de España 1991, 2001 y 2011 I (yps181 cesvima.upm.esire-habirbown)

e-cienciaDatos > Repositorio de Datos UPM > Catálogos de Barrios Vulnerables de España 1991, 2001 y 2011

\#. Contact 厄. Share

Los Catálogos de Barrios Vuinerables de España de 1991, 2001 y 2011 examinan la vulnerabilidad urbana en las ciudades españolas mayores de 50 mill habitantes y capitales de provincia para delimitaciones a escala de barrio elaboradas exprofeso. También analizan las concentraciones de población inmigrante en los años 2006 y 2011 . En total se analizaron 116 ciudades en 1991, 139 en 2001 y 2006, y 147 en 2011. Los Catálogos forman parte del Observatorio de la Vulnerabilidad Urbana (OVU) del Ministerio de Transportes, Movilidad y Agenda Urbana (MITMA) y sirven como punto de partida para el diseño de politicas públicas urbanas enmarcadas en sus competencias siguiendo el mandato de la Disposición Adicional Primera del Real Decreto Legislativo 7/2015, por el que se aprueba el Texto Refundido de la Ley de Suelo y Rehabilitación Urbana.

El objetivo de los Catálogos es localizar aquellos barrios en los que las condiciones de la población residente sean sensiblemente peores que las del conjunto de la población española. Los Catálogos constituyen una reflexión sobre la vulnerabilidad urbana desde una perspectiva urbanistica, centrada en la determinación y análisis de ámbítos concretos que se encuentran en peor situación que el resto de los tejidos urbanos españoles, y que pueden constituir piezas que por su tamaño, autonomía y homogeneidad puedan ser objeto de planes o programas de intervención que mejoren su posición. Los Catálogos ofrecen, además, una panorámica de la vulnerabilidad urbana de las ciudades españolas a lo largo de las dos décadas que abarcan, permitiendo analizar tanto los desequilibrios existentes en una ciudad, región o todo el pais, como su evolución en el periodo

Los resultados de los Catálogos son las delimitaciones de Barrios Vulnerables (BBVV), para los años 1991, 2001 y 2011; las delimitaciones de barrios con concentraciones significativas de población inmigrante (BBINM) en 2006 y 2011; y los indicadores estadisticos asociados a cada uno de los barrios mediante perimetros estadisticos, denominados en los Catálogos como Areas Estadisticas Vulnerables (AEV) y Áreas Estadisticas con concentraciones significativas de población inmigrante (AEINM). Todo ello se materializa en un conjunto de bases de datos, capas de información geográfica y un conjunto de fichas organizadas elaborado a partir de ambas.

En total se detectaron 370 Barrios Vulnerables en 81 ciudades en 1991, 621 Barrios Vulnerables en 103 ciudades en 2001, y 918 Barrios y Areas Estadisticas Vulnerables en 132 ciudades en 2011. Respecto a las concentraciones de población inmigrante, se detectaron 256 Barrios de Inmigración en 59 ciudades en 2006 . y 697 Barrios en 109 ciudades en 2011. La conclusión central de la investigación es que durante las dos décadas analizadas los desequilibrios internos de las ciudades españolas aumentaron de forma significativa y continuada, en un doble proceso de incremento y concentración espacial de población vulnerable.

I (xps181.cesvima unmes/re-hab/bbuv)

Search this dataverse....

\section{Author Name}

Gómez Giménez José Manuel (21)

Hernández Aia. Agustin (21)

Bodriguez Suárez.lván (21)

Subject

Social Sciences (21)

Keyword Term

Barrios de inmigración (21)

Barrios destavorecidos (21)

Barrios vuinerables (21)

Deprived neighbourhoods (21)

Urtan vulnerability_(21)

\begin{tabular}{|c|c|}
\hline \multicolumn{2}{|r|}{$\frac{\text { Catálogos de Barrios Vulnerables de España 1991.2001 y 2011. Bases de datos y capas de información gecgráfica }}{\text { Feb 2, } 2021}$} \\
\hline & 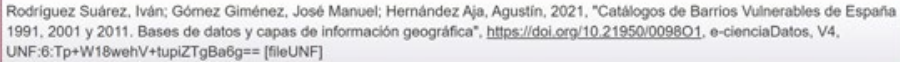 \\
\hline
\end{tabular}

Morem

Distribution Date

2021(20)

$$
\begin{aligned}
& \text { capas en formato geogr } \\
& \text { especificaciones de d.... }
\end{aligned}
$$

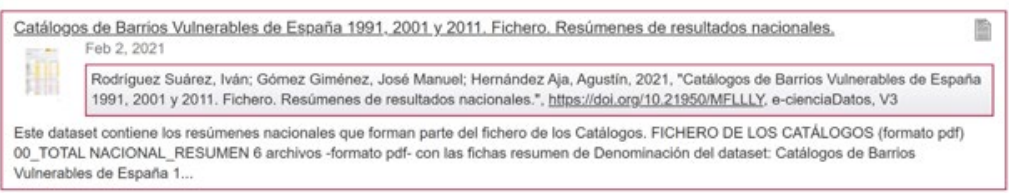
Do_TOTAL NACIONAL_RESUN
Vuinerables de Espana $1 . .$.

Catálogos de Barrios Vulnerables de España 1991. 2001 y 2011. Fichero. Comunidades Autónomas. 01 Andalucia

Rodriguez Suärez, Ivản; Gómez Giménez, José Manuel; Hernández Aja, Agustin, 2021, "Catálogos de Barrios Vulnerables de Espana 1991. 2001 y 2011. Fichero. Comunidades Autónomas. 01 Andalucla", https:/lidoi org/10.21950iCXU3VA. e-cienciaDatos, V3

Este dataset contiene toda la información del fichero de los Catálogos producida para Andalucia: los resúmenes autonómicos y los ficheros de ciudades en las que se detectaron barrios o dreas estadisticas en alguno de los anos. FICHERO DE LOS CATALLOGOS (formato pdf) 01_ANDALUCIAD.

FIG. 15/ Dataverse de los datos en abierto de los Catálogos de Barrios Vulnerables de España 1991, 2001 y 2011.

Fuente: página electrónica del repositorio E-CIENCIA. DATOS DEL PORTAL DE CIENCIA ABIERTA INVESTIGAM. https://edatos.consorciomadrono.es/dataverse/catalogos barrios vulnerables

Por último, desde 2021, los datos de los Catálogos de Barrios Vulnerables 1991, 2001 y 2011 han sido puestos a disposición pública y en abierto a través del repositorio de datos e-cienciaDatos del portal InvestigaM9 (FIG. 15).
A través de él, se puede acceder a todas las capas de información geográfica con las delimitaciones de Barrios y Áreas Estadísticas; a las bases de datos con todos sus indicadores numéricos y clasificatorios asociados, y a gran

\footnotetext{
${ }^{9}$ http://edatos.consorciomadrono.es/dataverse/catalogos
} barrios vulnerables 
cantidad de fichas con análisis detallados, organizadas por ciudades y comunidades autónomas; permitiéndose en todos los casos su libre descarga, utilización y edición.

En total, la información se compone de 471 archivos organizados en un dataverse, a su vez estructurado en veintiún datasets: uno de ellos para las bases de datos y capas de información geográfica, otro con información síntesis a nivel estatal y los otros diecinueve para los ficheros organizados por comunidades autónomas.

Para esta difusión se ha homogeneizado y ordenado toda la información de los Catálogos y se han producido fichas resumen (estatales, autonómicas y municipales), así como fichas de listados de Barrios y Áreas Estadísticas municipales. Además, en todos los datasets distribuidos se puede encontrar un documento síntesis con las especificaciones de los datos publicados donde ampliar información acerca de su contenido y de la metodología empleada (RODRíGUEZ \& al., 2021a y 2021b).

\section{Conclusiones}

\subsection{Características y utilidad}

Los Catálogos de Barrios Vulnerables ofrecen una amplia panorámica sobre la vulnerabilidad urbana en las ciudades españolas que abre la reflexión sobre sus posibles causas y la forma en la que puede remediarse. Las tres ediciones de los Catálogos describen las condiciones sociodemográficas y de calidad del alojamiento de los barrios y áreas vulnerables delimitados. Su objetivo es caracterizar la vulnerabilidad urbana desde la óptica de la calidad de vida urbana, estableciendo la necesidad de identificar los problemas y las oportunidades de estos barrios, adelantándose de alguna manera al concepto de resiliencia urbana, y estableciendo como prioridad la necesidad de dotar a esas áreas y a sus habitantes de las condiciones necesarias para mantener o recuperar su condición de ciudad y ciudadanía. Para ello es necesario conocer la realidad de nuestras ciudades, que es la misión de los Catálogos, de forma que a través de este conocimiento se desarrollen políticas públicas que integren las múltiples dimensiones de la calidad de vida urbana. Los Catálogos son, por tanto, herramientas útiles para determinar el fenómeno de la vulnerabilidad urbana en España y para el desarrollo de la regeneración urbana integrada, gracias a la información elaborada y a la delimitación urbanística de los barrios que contienen. Los contenidos de los Catálogos se pueden dividir en dos grandes paquetes de aportaciones: el referido a la utilidad de la información de carácter sociodemográfico y de su cuerpo de estudio, que incluye el rango temporal y la determinación de las ciudades estudiadas; y el urbanístico, que supone, junto con la concepción de la calidad de vida urbana como constructo multidimensional, una aportación fundamental de los Catálogos, al señalar espacios aptos para una intervención urbana integral.

Utilidad de la información elaborada y del cuerpo de estudio:

- Dimensión temporal: análisis a lo largo de dos décadas, con tres cortes temporales (1991, 2001 y 2011) para los indicadores de vulnerabilidad y dos (2006 y 2011) para el indicador de concentraciones de población inmigrante.

- Rango de ciudades: el trabajo se extiende a todas las ciudades mayores de 50.000 habitantes y las capitales de provincia en cada uno de los cortes temporales. Los Catálogos son, por tanto, un análisis amplio de la realidad urbana española.

- Información sobre el barrio y su ciudad: la información elaborada se presenta con el mismo detalle para todas las ciudades en las que se detectaron barrios y para cada uno de ellos se ofrece información estadística con la misma desagregación.

- Facilidad de interpretación de los resultados: al utilizar indicadores directos elaborados a partir de variables simples y fácilmente comunicables, se pretende que puedan ser utilizados como herramienta de comunicación con las poblaciones afectadas y como base para el seguimiento y evaluación de los proyectos desarrollados.

- Estabilidad en las dimensiones de la vulnerabilidad analizadas: a pesar de los cambios metodológicos de uno de los indicadores, derivados de la desaparición o modificación de las variables de cada Censo, los indicadores utilizados se han adaptado a estos cambios, buscando permitir la comparación entre ellos y, por tanto, analizar la evolución de la vulnerabilidad en cada ciudad o comunidad autónoma.

- Umbrales de referencia estatales para el análisis de la vulnerabilidad: los Catálogos han optado por la utilización de valores de referencia nacionales, que pueden 
necesitar de ajustes locales para el análisis de una única ciudad, pero que por el contrario permiten comparar y evaluar la situación y evolución entre las diferentes regiones y áreas urbanas del país y por tanto son útiles para la realización de estudios sobre la vulnerabilidad y su evolución a diversas escalas: estatal, regional o de ciudad.

- Utilización de un sistema de clasificación flexible que permite incorporar otros indicadores relacionados con otras dimensiones de la vulnerabilidad, propios de cada ciudad o en función de las áreas de investigación que se incorporen. También permiten analizar la evolución de los barrios de una misma ciudad o comparar distintas ciudades al utilizar los valores nacionales como referencia, presentando ventajas para la comunicación con las poblaciones afectadas, al ofrecer sus resultados en cuatro grados fácilmente comprensibles.

Ventajas de la utilización de delimitaciones urbanísticas:

- Un mayor ajuste a la realidad urbana: las agregaciones espaciales de barrio superan las limitaciones de las secciones censales. Las secciones censales tienen como finalidad la organización de los procesos electorales, y se consideran como el nivel mínimo para la provisión de datos estadísticos que no supongan una vulneración de la protección de datos personales. En cualquier caso, se utilizan en carencia de otras delimitaciones, como la base territorial para las investigaciones estadísticas, ya que permiten acceder a través de ellas de los datos del Padrón Municipal de Habitantes y los Censos de Población y Viviendas. Se trata, por tanto, de delimitaciones independientes de la realidad de las estructuras urbanas (aunque no son totalmente ajenas a ella), por lo que resulta necesario asociar sus datos a aquellos espacios urbanos que las contienen o intersecan con ellas.

- Delimitación de Barios Urbanísticos: La realidad de la intervención necesita de áreas urbanas con cierta homogeneidad, social y morfológica, y diferenciación en la trama urbana que tengan el tamaño suficiente como para permitir intervenciones en las que sea posible aplicar políticas urbanas integrales. El tamaño y características de estas delimitaciones convierten estos espacios en ámbitos con potencial de transformación urbana sobre los que desarrollar planes, programas y proyectos de actuación de dimensión urbana que contribuyan al reequilibrio territorial.
Se trata de espacios que, en muchos casos, tienen tamaño suficiente para acoger todas las funciones de la vida cotidiana y en las que éstas se pueden desarrollar en proximidad. Además, el barrio es un marco con capacidad de interacción y autonomía en interdependencia con la ciudad y la metrópoli (AlguACIL, 1998).

\subsection{Limitaciones}

Las principales limitaciones de los Catálogos están relacionadas con las propias limitaciones derivadas de la utilización de los Censos como fuente de datos, la amplitud del periodo temporal de elaboración de los Catálogos, los municipios objeto de estudio, los criterios para la delimitación urbanística de los barrios y las disfunciones en la difusión de la información generada.

La utilización del Censo como base del análisis limita el análisis a una vez cada diez años y somete el trabajo a las limitaciones de su propia metodología. En el caso del Censo de 2011, la falta de disponibilidad, en algunos casos, de información desagregada por sección censal, y los problemas de fiabilidad de algunas variables, especialmente las referidas a grupos pequeños, motivaron cambios metodológicos significativos en el Catálogo de 2011 para permitir mantener la estructura de los anteriores y poder comparar su evolución. Es previsible que se produzcan nuevos cambios en la elaboración del Censo de Población y Vivienda, que obliguen a nuevos ajustes metodológicos en las actualizaciones de los Catálogos.

La necesidad de que la metodología de análisis sea estable a lo largo de la existencia de los Catálogos, dos décadas para los tres realizados, y la decisión de buscar una homogeneidad en su estructura que permita la comparación y el análisis de la evolución de la vulnerabilidad, dificulta que pueda abordarse la introducción de otras dimensiones de la vulnerabilidad que puedan resultar más adecuadas para reflejar la realidad cambiante de las estructuras urbanas y la población que las habita.

En ocasiones la limitación de los municipios analizados a contener un mínimo de 50.000 habitantes impide disponer de una completa visión metropolitana en el análisis de los procesos de segregación urbana, por ello es necesaria una reflexión sobre los objetos de análisis que hasta la fecha ha elegido el Catálogo como base de sus trabajos. La evolución de las fracturas 
socioeconómicas y los cambios en la segregación espacial de los territorios urbanos hacen recomendable la realización de estos trabajos a la escala real en la que operan ciudadanos y agentes económicos: la del continuo urbano funcional, más allá de su fragmentación municipal y del tamaño poblacional de estos.

Además, más allá de cuál sea el objeto de análisis, municipios o áreas urbanas funcionales, los criterios poblacionales mínimos y máximos para la delimitación de los Barrios Vulnerables también podrían ser repensados. Los cambios espaciales en las unidades estadísticas de las que disponemos (las secciones censales) introducen serias dificultades a la hora de realizar el seguimiento de los indicadores de vulnerabilidad de las áreas catalogadas con los datos de las diferentes ediciones del Censo de Población y Viviendas. Además, aunque los criterios de delimitación de los barrios presentan una horquiIla suficientemente amplia (entre 3.500 y 15.000 habitantes), se ha comprobado que existen casos en los que es conveniente sobrepasarla. El problema aparece generalmente en unidades de tamaño poblacional por debajo del límite inferior. Aunque la población vulnerable se halla mayoritariamente concentrada en barrios, en el Catálogo de 2011 se contabilizaron hasta 34 ciudades en las que la población vulnerable dispersa en unidades menores era superior a la existente en los barrios delimitados con los criterios demográficos exigidos. La mayor parte de estas ciudades se caracterizan por su pequeño tamaño o por tener una configuración de modos de urbanización tradicional extendida (la aldea gallega o la suburbanización de la huerta murciana). En estos casos en que la urbanización funcional no se limita al continuo urbano, parece necesario buscar una alternativa para que el Catálogo pueda acoger este tipo de barriadas y desarrollos urbanísticos de menor tamaño.

Por otro lado, se han constatado hasta ahora ciertas debilidades en la difusión de la información de los Catálogos. Se trata de una información extensa elaborada en un periodo temporal amplio. Esto ha ocasionado que la difusión no se haya podido realizar de forma tan sistemática como sería deseable. El primer Catálogo de 1991 se produjo en papel, la utilización de herramientas informáticas era aún incipiente y el uso de internet minoritario. Con el Catálogo de 2001 se introdujo la difusión a través de internet

\footnotetext{
$10 \mathrm{http}: / /$ edatos.consorciomadrono.es/dataverse/catalogos barrios vulnerables
}

y se digitalizaron los contenidos del de 1991. El Catálogo de 2001 resulta el más completo ya que utilizó herramientas SIG para su elaboración, sobre los datos universales del Censo y manteniendo el trabajo de delimitación urbanística que incluía la visita a las ciudades estudiadas. El Catálogo de 2011, aunque aprovechó las ventajas de las mejoras de los medios informáticos, se realizó con los datos del Censo de 2011, que ya no procedían de una encuesta universal por lo que, aunque coherentes desde el punto de vista estadístico, demostraron problemas en las escalas menores. El Catálogo de 2011 tuvo que renunciar a la delimitación urbanística por limitaciones presupuestarias. Aun así, desde su puesta en funcionamiento, ha sido posible consultar la información de los tres Catálogos a través del Visor de Barrios Vulnerables del Ministerio, que recoge parte de la información de los Catálogos. Por otro lado, desde este año también es posible descargar su información en formatos reutilizables a través del repositorio ecienciaDatos ${ }^{10}$. Aunque se ha dilatado demasiado esta posibilidad, con ello se espera que mejore la difusión y utilización de los Catálogos (RODRÍGUEZ \& al., 2021a).

\subsection{Perspectivas de futuro}

Son tres los retos más importantes a los que se enfrenta la continuidad de los Catálogos: el primero relacionado con los contenidos del Censo de Población y Vivienda 2021, el segundo con continuar mejorando la accesibilidad a la información producida y su difusión, y el último con desarrollar las posibilidades para que los Catálogos puedan ser utilizados como herramientas de apoyo para el diagnóstico y evaluación de políticas públicas urbanas.

El próximo Censo 2021 permitirá actualizar el Catálogo con una cuarta edición y disponer de un nuevo corte temporal que amplíe el análisis a las tres últimas décadas, que permitirá tanto nuevas investigaciones como su utilización para el diseño y evaluación de políticas públicas. El reto del Censo de 2021 es que su metodología prescindirá por completo del trabajo de campo y se desarrollará en su totalidad a partir de registros administrativos ${ }^{11}$. Aunque esto puede suponer una limitación de la información disponible para la escala de la sección censal, es

\footnotetext{
${ }^{11}$ Información detallada sobre la metodología del futuro Censo 2021 puede ser consultada en la página electrónica del INE: https://www.ine.es/censos2021/
} 
previsible que los resultados definitivos estén disponibles antes que en los Censos anteriores, lo que puede ayudar a que el contenido del Catálogo esté más ajustado a la realidad de los espacios analizados. El cambio metodológico conllevará la revisión de la metodología del futuro Catálogo, que deberá permitir la comparabilidad con los análisis de los anteriores, y por tanto el análisis de la evolución de la vulnerabilidad en España.

Las posibilidades de mejora en la difusión de la información producida para los Catálogos son aún amplias. La reciente puesta a disposición pública de los perímetros delimitados y las bases de datos asociadas en formatos reutilizables, ajustando los Catálogos a los principios generales de publicidad activa recogidos en la Ley 19/2013, de 9 de diciembre, de transparencia, acceso a la información pública y buen gobierno (JEFATURA DEL ESTADO, 2013b), puede suponer un hito a este respecto, pero aún es pronto para evaluar sus repercusiones. A partir de ahora, la tarea pendiente debería ser continuar mejorando la utilidad de la información, algo que permitirá e incentivará la producción de trabajos complementarios a los propios $\mathrm{Ca}$ tálogos.

Por último, sería deseable que se incrementara el uso de los Catálogos como herramienta de apoyo al diagnóstico de áreas necesitadas de intervención, o para la evaluación y acompañamiento de políticas públicas. Los Catálogos están siendo utilizados en distintos planes y proyectos como en la Agenda Urbana Española o en las Estrategias de Desarrollo Urbano Sostenible e Integrado (EDUSI), y se han utilizado en diversos instrumentos de planeamiento o diagnóstico municipal, aunque no siempre se menciona de forma explícita el Catálogo, como puede ser el caso de las políticas estatales de vivienda, en las que se exige una memoriaprograma para la declaración de áreas de rehabilitación y regeneración urbana que reciben financiación pública y en las que podría resultar de gran interés su utilización.

\section{Bibliografía}

ARIAS GoYTRE, F. (director) (2000): La desigualdad urbana en España. Madrid, Ministerio de Fomento, Centro de Publicaciones. http://habitat.aq.upm.es/ due/

AlguACIL Gómez, J. (1998): Calidad de Vida y Praxis Urbana. Nuevas iniciativas de gestión ciudadana en la periferia social de Madrid. CIUDADES POR UN
FUtURO MÁS SOSTENIBLE CF+s. http://habitat. aq.upm.es/cvpu/

de Santiago Rodríguez, E. (2018): Herramientas de diagnóstico para las intervenciones de regeneración urbana integrada en la ciudad consolidada: ejemplos de España. Limaq, (004), 219-246. https:// doi.org/10.26439/limaq2018.n004.2658

JefATURA DEL EstAdo (2013a): Ley 8/2013, de 26 de junio, de rehabilitación, regeneración y renovación urbanas. Boletín Oficial del Estado 27/06/2013. https://www.boe.es/eli/es///2013/06/26/8

- (2013b): Ley 19/2013, de 9 de diciembre, de transparencia, acceso a la información pública y buen gobierno. Boletín Oficial del Estado 10/12/2013.

https://www.boe.es/eli/es///2013/12/09/19/con

HeRnÁNDEZ AJA, A. \& al., (2013): Análisis de las características de la edificación residencial en España en 2001. A nivel nacional y por comunidad autónoma. Madrid, España, Ministerio de Fomento e Instituto Juan de Herrera de la Universidad Politécnica de Madrid.

https://www.mitma.gob.es/recursos mfom/ pdf/9DFC55DD-B6F8-4C40-8634-8B199A6770A8 1135887/INF CARAC EDIF RESID 2001.pdf

- \& al., (2014): Análisis de las características de la edificación residencial en España en 2011. A nivel nacional y por comunidad autónoma. Madrid, España, Ministerio de Fomento e Instituto Juan de Herrera de la Universidad Politécnica de Madrid. http://oa.upm.es/52769

- \& al., (2015): Atlas de Barrios Vulnerables de España: 12 Ciudades 1991/2001/2006. Madrid, España, Instituto Juan de Herrera de la Universidad Politécnica de Madrid. ISBN 978-84-9728-518-6. http://oa.upm.es/34999

- \& al., (2018a): Fórmulas innovadoras de gestión y financiación en actuaciones de regeneración de barrios. Madrid, España, Ministerio de Fomento e Instituto Juan de Herrera de la Universidad Politécnica de Madrid. http://oa.upm.es/54607

— \& al., (2018b): Barrios vulnerables de las grandes ciudades españolas. 1991/2001/2011. Madrid, España, Instituto Juan de Herrera de la Universidad Politécnica de Madrid. ISBN 978-84-9728-568-1. http://oa.upm.es/51015

- \& al., (2018c): Vulnerabilidad residencial y social en las grandes ciudades españolas. 2001 / 2011. Madrid, España, Instituto Juan de Herrera de la Universidad Politécnica de Madrid. ISBN 978-849728-569-8. http://oa.upm.es/51018

MINISTERIO DE FOMENTO (2015): Real Decreto Legislativo $7 / 2015$, de 30 de octubre, por el que se aprueba el texto refundido de la Ley de Suelo y Rehabilitación Urbana. Boletín Oficial del Estado 31/10/2015. https://www.boe.es/eli/es/rdlg/2015/10/30/7/con

- (2016): Síntesis Metodológica general del Catálogo de Barrios Vulnerables e Indicadores Básicos de Vulnerabilidad Urbana. Ministerio de Fomento e Instituto Juan de Herrera de la Universidad Politécnica de Madrid.

https://portalweb2.fomento.gob.es/Barrios Vulnerables 
- (2019): Agenda Urbana Española. https://www.aue. gob.es/

Ministerio DE TRANSPORTES, MOVILIDAD Y AgENDA URBANA (2020): Observatorio de la Vulnerabilidad Urbana.

https://www.mitma.gob.es/arquitectura-vivienda-ysuelo/urbanismo-y-politica-de-suelo/observatoriode-la-vulnerabilidad-urbana

MINISTERIO DE LA PRESIDENCIA, RELACIONES CON LAS CORTES Y MEMORIA DEMOCRÁtICA (2020): Resolución de 29 de abril de 2020, de la Subsecretaría, por la que se publica la Resolución de 17 de febrero de 2020, de la Presidencia del Instituto Nacional de Estadística y de la Dirección General de Cooperación Autonómica y Local, por la que se dictan instrucciones técnicas a los Ayuntamientos sobre la gestión del Padrón municipal. Boletín Oficial del Estado 02/05/2020. https://www.boe.es/eli/es/res/2020/04/29/(5)/con

OCDE (1998): Integrating Distressed Urban Areas. Final report, Urban Affairs Division, OCDE Paris. ISBN 978-92-64-16062-0.

Rodríguez SuAREZ, I. \& al., (2021a): Dataverse de los Catálogos de Barrios Vulnerables de España 1991, 2001 y 2011. e-cienciaDatos.

https://edatos.consorciomadrono.es/dataverse/ catalogos barrios vulnerables

- \& al., (2021b): Datos abiertos de los Catálogos de Barrios Vulnerables de España 1991, 2001 y 2011. Especificaciones de los datos publicados en el repositorio e-cienciaDatos del Consorcio Madroño. Madrid: Universidad Politécnica de Madrid. http://oa.upm.es/65954/

UE (2013): Reglamento N. ${ }^{\circ} 1301 / 2013$ sobre el Fondo Europeo de Desarrollo Regional (2014-2020). EUR-Lex. Access to European Union Law. https://eur-lex.europa.eu/legal-content/ES/ LSU/?uri=CELEX\%3A32013R1301

\section{Listado de acrónimos/siglas}

AEV:

BV / BBVV:

$\mathrm{CF}+\mathrm{S}$ :

EDUSI:

Área Estadística Vulnerable.

Barrio/s Vulnerable/s.

Ciudades para un futuro más sostenibles.

Estrategias de Desarrollo Urbano Sostenible e Integrado.

IBVU: Indicador Básico de Vulnerabilidad Urbana.

IEST:

IJH:

IPAR:

INE:

IVIV:

IVIV1:

Indicador de estudios.

Instituto Juan de Herrera.

Indicador de paro.

Instituto Nacional de Estadística.

Indicador de vivienda.

IVIV2: $\quad$ Segundo indicador de vivienda (IVIV) empleado en 1991.

IVIV3: $\quad$ Tercer indicador de vivienda (IVIV) empleado en 1991.

MN: Media Nacional.

OCDE: $\quad$ Organización para la Cooperación y el Desarrollo Económicos.

OVU: Observatorio de la Vulnerabilidad Urbana.

SIG: $\quad$ Sistemas de Información Geográfica.

VC: Vulnerabilidad crítica.

VS: $\quad$ Vulnerabilidad severa.

V: $\quad$ Vulnerabilidad media.

VL: $\quad$ Vulnerabilidad leve.

VR: Valor de Referencia. 